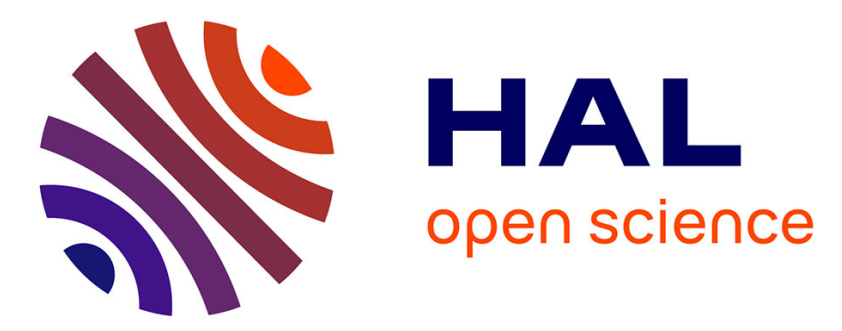

\title{
Bounded motion design in the Earth zonal problem using differential algebra based normal form methods
}

\author{
Adrian Weisskopf, Roberto Armellin, Martin Berz
}

\section{To cite this version:}

Adrian Weisskopf, Roberto Armellin, Martin Berz. Bounded motion design in the Earth zonal problem using differential algebra based normal form methods. Celestial Mechanics and Dynamical Astronomy, 2020, 132 (14), pp.1-32. 10.1007/s10569-020-9953-x . hal-03100444

\section{HAL Id: hal-03100444 \\ https://hal.science/hal-03100444}

Submitted on 6 Jan 2021

HAL is a multi-disciplinary open access archive for the deposit and dissemination of scientific research documents, whether they are published or not. The documents may come from teaching and research institutions in France or abroad, or from public or private research centers.
L'archive ouverte pluridisciplinaire $\mathbf{H A L}$, est destinée au dépôt et à la diffusion de documents scientifiques de niveau recherche, publiés ou non, émanant des établissements d'enseignement et de recherche français ou étrangers, des laboratoires publics ou privés. 


\section{OATAO \\ Open Archive Toulouse Archive Ouverte}

\section{Open Archive Toulouse Archive Ouverte (OATAO)}

OATAO is an open access repository that collects the work of some Toulouse researchers and makes it freely available over the web where possible.

This is an author's version published in: https://oatao.univ-toulouse.fr/26950

Official URL:https://doi.org/10.1007/s10569-020-9953-x

\section{To cite this version :}

Weisskopf, Adrian and Armellin, Roberto and Berz, Martin Bounded motion design in the Earth zonal problem using differential algebra based normal form methods. (2020) Celestial Mechanics and Dynamical Astronomy, 132 (14). 1-32. ISSN 0923-2958

Any correspondence concerning this service should be sent to the repository administrator: 


\title{
Bounded motion design in the Earth zonal problem using differential algebra based normal form methods
}

\author{
A. Weisskopf ${ }^{1}$ (D) R. Armellin ${ }^{2} \cdot$ M. Berz ${ }^{1}$
}

\begin{abstract}
Establishing long-term relative bounded motion between orbits in perturbed dynamics is a key challenge in astrodynamics to enable cluster flight with minimum propellant expenditure. In this work, we present an approach that allows for the design of long-term relative bounded motion considering a zonal gravitational model. Entire sets of orbits are obtained via highorder Taylor expansions of Poincarè return maps about reference fixed points. The high-order normal form algorithm is used to determine a change in expansion variables of the map into normal form space, in which the phase space behavior is circular and can be easily parameterized by action-angle coordinates. The action-angle representation of the normal form coordinates is then used to parameterize the original Poincarè return map and average it over a full phase space revolution by a path integral along the angle parameterization. As a result, the averaged nodal period and drift in the ascending node are obtained, for which the bounded motion conditions are straightforwardly imposed. Sets of highly accurate bounded orbits are obtained, extending over several thousand kilometers, and valid for decades.
\end{abstract}

Keywords Normal form methods · Bounded motion · Zonal problem · Differential algebra

\section{Introduction}

Henri Poincaré's three volumes on 'New Methods of Celestial Mechanics' (Poincaré 1899) were one of the greatest methodological contributions not only to the field of celestial mechanics, but to the mathematical theory of dynamical systems in general. Based on his work, numerous methods to analyze dynamical systems have been established and developed in

$凶$ A. Weisskopf

weisskop@msu.edu

R. Armellin

roberto.armellin@isae-supaero.fr

M. Berz

berz@msu.edu

1 Michigan State University, East Lansing, Michigan 48824, USA

2 Department of Aerospace Vehicles Design and Control-DCAS, Institut Supérieur de l'Aéronautique et de l'Espace (ISAE-SUPAERO), 31055 Toulouse, France 
various fields. Due to their common origin and mathematical underpinnings, it is sometimes possible to transfer specific methods from one field of dynamical systems to another. In this paper, we transfer differential algebra (DA)-based normal form methods, which were inspired by Poincaré work and first developed in the field of particle beam physics and accelerator physics, to the field of astrodynamics to design bounded motion in the Earth's zonal problem.

The DA framework (Berz 1987, 1988), the DA normal form algorithm (Berz 1993, 1999) and their associated techniques are hybrid methods of numerical and analytic calculations and have been established by Berz et al. Many of the methods use concepts going back to Poincaré (1899), especially considering his work on perturbation theory, return maps (later: Poincaré maps), non-integrability and integral invariants. While the DA methods are derived on a general basis, they have predominantly been applied and developed in the field of accelerator physics, where they reveal details of those dynamical systems that are otherwise very difficult to obtain by conventional methods. More recently, researchers have begun on the fruitful transfer of those DA methods to the astrodynamical community (Lizia et al. 2008; Armellin et al. 2010a, b; Wittig et al. 2015). An advancement of this transfer to normal form methods provides new possibilities, as it will be demonstrated in this work, including the capability of determining entire sets of bounded motion orbits in the zonal problem.

Two or more objects are in bounded motion if the relative distance between them remains bounded for an extended period of time. In practice, this finds application in cluster flight (Brown and Eremenko 2006) and formation flying (Alfriend et al. 2010) missions, which can offer many advantages compared to single spacecraft mission. From the scientific standpoint, they enable measurements of unprecedented spatial and temporal correlation, but they also have economic advantages such as allowing for redundancies within the group, a distribution of the payload and the adaptability of the mission by exchanging modules of the group. Missions such as PRISMA (D'Amico et al. 2012), GRACE (Montenbruck et al. 2006) and TerraSAR-X and TanDEM-X (D'Amico and Montenbruck 2006) demonstrated the practicability of formation flying and stimulated further research in the field.

To minimize the amount and extend of formation-keeping maneuvers with control strategies during the mission, it is of great interest to the astrodynamical community to find 'naturally' bounded motion orbits for models considering as many perturbations as possible, which leave only the unmodeled perturbations to be corrected by control maneuvers.

In Keplerian dynamics, bounded motion is obtained by choosing orbits with the same semimajor axis, i.e., orbits with the same orbit period and energy. The most general expression of the energy-matching condition for Keplerian orbits in Euler-Hill coordinates was derived by Gurfil (2005). Alternatively, the linearized relative equations of motion (Clohessy and Wiltshire 1960; Sullivan et al. 2017) and Tschauner-Hempel equations (Inalhan et al. 2002) can be used to determine initial conditions which produce periodic relative orbits.

Introducing perturbations to the dynamics generates a non-trivial bounded motion problem. The dominating perturbation is often due to the oblateness of the central body and the associated zonal perturbation from the second zonal harmonic coefficient $J_{2}$ of the gravitational potential. This zonal perturbation introduces a drift in the right ascension of the ascending node (RAAN), the argument of periapsis and the mean anomaly. The drift in each of the quantities is oscillating at different frequencies, which drastically increases the complexity of the bounded motion problem.

Two main routes have been followed to approach this problem: The first one is based on analytic derivations and the second one uses fully numerical techniques. The analytic approaches were mainly developed in reduced zonal problems, e.g., considering $J_{2}$ only. Schaub and Alfriend (2001) derived conditions for the first-order differential mean orbit elements, which identify $J_{2}$-invariant orbits by minimizing the relative drift in the RAAN and 
mean argument of latitude. The resulting relative motion remains bounded for short times and short relative distances due to the unmodeled nonlinear effects and short-periodic oscillations. Further linear approaches (Gim and Alfriend 2003; Schweighart and Sedwick 2002) using a state transition matrix and approaches using non-osculating orbital elements (Gurfil 2007; Dang et al. 2015) offered a deeper understanding of the mathematical underpinnings of the bounded motion problem, while yielding similarly limited results in size and duration. The approach by Chu et al. (2015) within the framework of analytical theory used HamiltonJacobi theory and action-angle variables to derive canonical solutions for a constant nodal period and drift of the ascending node for the pseudo-elliptical orbits and matching those constant values between pairs of orbits.

On the other hand, numerical approaches enabled the calculation of bounded motion conditions in a more complete dynamical model, thus avoiding the inaccuracies introduced in the osculating to mean transformations and by linearization. The pioneering work by Broucke (1994) on families of two-dimensional quasi-periodic invariant tori around stable periodic orbits of the Ruth-reduced axially symmetric system, was used by Koon et al. (2001) in combination with Poincaré section techniques to study the $J_{2}$ problem. While this method was an improvement to the first-order approaches, long-term bounded motion was still not achieved by placing orbits on the center manifold. Xu et al. (2012) pointed out that long-term bounded motion in the zonally perturbed system could only be achieved when the RAAN drift and nodal period are on average the same for each of the bounded modules. These constrains are weaker than the constrains originally derived by Martinusi and Gurfil (2011), and when they are enforced on a numerical searching method based on ergodic maps and contour plots, the resulting relative motion is bounded for more than two years.

In Baresi and Scheeres (2017a), a fully numerical technique based on stroboscopic maps was used to obtain entire families of quasi-periodic orbits producing bounded relative motion about a periodic one. This method was then used to study both bounded motion about asteroids (Baresi et al. 2018) and in low Earth, medium Earth and geostationary orbits (Baresi and Scheeres 2017b). Numerical approaches yield bounded relative orbits with arbitrary size over very long periods of time (or infinite time in theory). However, they require complex and time-consuming algorithms.

In He et al. (2018), a compromise between the analytic and numerical technique was presented, based on the use of DA. DA techniques were used to expand high-order mapping between two consecutive equatorial crossings (i.e., Poincaré maps). This enabled the study of the motion of a spacecraft for many revolutions by the fast evaluation of Taylor polynomials. The problem of designing bounded motion orbits was then reduced to the solution of two polynomial nonlinear equations (constraining the mean nodal period, $T_{\mathrm{d}}$, and drift of the right ascension of the ascending node, $\Delta \Omega$ ). The derived method showed an accuracy comparable with that of fully numerical methods, but with a reduced complexity due to the introduced polynomial approximations. The main drawback of this technique consisted in the calculation of the mean $T_{\mathrm{d}}$ and $\Delta \Omega$ using numerical averaging over thousands of nodal crossings. This process resulted in the main computationally intensive part of the algorithm and was also responsible for accuracy degradation in case of very large separations.

The aim of this paper is to overcome this limitation by the introduction of DA-based normal form (DANF) methods. In particular, the DANF algorithm is used to determine a change in expansion variables of the Poincare map into normal form space, where the phase space behavior is circular and can be easily parameterized by action-angle coordinates (Fig. 1). The parameterized set of normal form phase space coordinates is then transformed back to the original coordinates and averaged over a full phase space revolution by a path integral along the angle parameterization, yielding the Taylor expansion of the averaged bounded motion 
quantities $T_{\mathrm{d}}$ and $\Delta \Omega$. The expansions are transformed back to the original coordinates. Map inversion methods are then used to match two remaining expansion variables in the constants of motion such that the bounded motion conditions are met. As a result, an entire set of bounded orbits extending up to thousands of kilometers with a stable relative motion for decades is achieved in the full zonal problem, avoiding the numerical averaging introduced in He et al. (2018). The superiority in terms of elegance, computational time and accuracy of the new algorithm will be demonstrated using similar test cases to those presented in $\mathrm{He}$ et al. (2018) and Baresi and Scheeres (2017b).

The paper is organized as follows. Firstly, we introduce the DA framework, the associated DA Poincaré maps and the DA normal form algorithm, with an example. Secondly, we discuss the zonal problem and bounded motion conditions, before moving on to the calculation of DA Poincaré return maps. Lastly, we present parameterized sets of bounded orbits for a low Earth orbit example from He et al. (2018) and a medium Earth orbit example from Baresi and Scheeres (2017b) before discussing the boundaries of the DANF method, which are far beyond any realistic/practical setup.

\section{Methods}

The methods in this paper are hybrids of numerical and analytic calculations and are based on a DA framework which was first developed to its current extent by Berz (1999, 1987, 1988). The following summary and introduction to the DA framework, DA maps and the DANF algorithm is based on Berz (1999) and was first given in Weisskopf et al. (2019).

\subsection{Differential algebra framework}

The basic goal of the DA framework is the representation and manipulation of analytic functions. To standardize the notation, an analytic function $f$ is expressed up to order $m$ in terms of its Taylor polynomial expansion $\mathcal{T}_{f}$, similar to how real numbers are approximated to a certain arbitrary number of significant digits. Instead of just using ' $\approx$ ' to represent the approximation, the notation ' $={ }_{m}$ ' is used to express that both sides are equivalent up to order $m$. Because of the approximation, multiple functions may be represented by the same Taylor polynomial of order $m$ and are therefore equivalent up to that order $\left('={ }_{m}\right.$ ').

This gives rise to the definition of equivalence classes following Berz (1999, p. 91). The equivalence class $[f]_{m}$ represents all elements $f$ of the vector space of infinitely differentiable functions $\mathcal{C}^{\infty}\left(\mathbb{R}^{n}\right)$ with $n$ real variables that have identical derivatives at the origin up to order $m$. The origin is chosen out of convenience and without loss of generality-any other point may be selected. In the DA framework, the equivalence class $[f]_{m}$ is represented by a DA vector, which stores all the coefficients of the Taylor expansion of $f$ and the corresponding order of the terms in an orderly fashion. Operations are now defined on the vector space ${ }_{m} D_{n}$ of all the equivalence classes []$_{m}$, where $n$ is the number of variables.

There are three operations: addition, vector multiplication and scalar multiplication, which are equivalent to the truncated result of adding two polynomials, multiplying two polynomials and multiplying them with a scalar. The first two operations on the equivalence classes (DA vectors) form a ring, and the scalar multiplication makes the three operations on the real (or complex) DA vectors an algebra, where not every element has a multiplicative inverse. An intuitive example of such elements is functions expanded at zero without a constant part. To make the algebra a differential algebra, the derivation $D$ satisfying Leibniz's law $(D(f g)=$ 
$f D(g)+g D(f))$ is introduced, which is almost trivial in the picture of differentiating polynomial expansions. The derivation opens the door to algebraic treatment of ordinary and partial differential equations as it is common in the study of differential algebras (Ritt and Liouville 1948; Ritt 1932; Kolchin 1973).

Implemented in COSY Infinity (Berz and Makino 2017; Makino and Berz 2006), the DA framework allows preserving the algebraic structure up to arbitrary order while manipulating the coefficients of the DA vectors with floating-point accuracy. An example of a DA vector in the application of DA transfer maps and Poincaré maps is given in Sect. 2.2.

\subsection{DA transfer maps and Poincaré maps}

Transfer maps are a standard tool in dynamical system theory to represent the effect of the flow generated by a set of ordinary differential equations (ODE). They are also called propagators or simply maps. Basically, a transfer map $\mathcal{M}$ algebraically expresses how a final state $\mathbf{z}_{f}$ is dependent on an initial state $\mathbf{z}_{i}$ and the system parameters $\boldsymbol{\eta}, \mathbf{z}_{f}=\mathcal{M}\left(\mathbf{z}_{i}, \eta\right)$.

In the DA framework, this is implemented by a local expansion of $\mathcal{M}$ in $(\delta \mathbf{z}, \delta \boldsymbol{\eta})$ up to order $m$ around an expansion point $\mathbf{z}_{0}$ and a reference set of parameters $\boldsymbol{\eta}_{0}$. The expansion point of the map belongs to a chosen reference orbit of the system, e.g., a (pseudo-)closed orbit for a fixed-point map and/or the ideal orbit of the unperturbed system.

There are special transfer maps, called Poincaré return maps or Poincaré maps for short, that constrain the initial and final state to the same Poincaré surface $\mathbb{S}$, reducing the dimension of the original map. These maps are particularly useful for repetitive systems, where multiple applications of the Poincare return map correspond to a propagation of the system. System dynamics represented by a Poincaré return maps can be further analyzed by normal form methods and for the asymptotic stability of the system.

Constraining the map to a surface is often done by calculating the flow of an ODE and projecting the flow onto the Poincaré surface to generate the Poincaré map. The DA Poincaré projection itself makes use of DA inversion methods that compute the inverse $\mathcal{A}^{-1}$ to the auxiliary map $\mathcal{A}$, which contains the constraining conditions of the Poincare surface, such that $\mathcal{A}^{-1} \circ \mathcal{A}={ }_{m} \mathcal{A} \circ \mathcal{A}^{-1}={ }_{m} \mathcal{I}$, given that $\mathcal{A}$ has no constant part. The basic idea of the projection of a transfer map $\mathcal{M}$ onto a surface defined by $\sigma(\mathbf{z}, \boldsymbol{\eta})=0$ is to replace one of the variables or parameters of $\mathcal{M}$ by an expression in terms of all the other variables and parameters such that the constraint $\sigma(\mathcal{M})=0$ is satisfied. This eliminates the component of the map and thereby reduces its dimensionality. An implementation of a timewise projection is outlined in Grote et al. (2006), where the map $\mathcal{M}$ is expanded in the independent variable time $t$ to find the intersection time $t^{\star}(\mathbf{z}, \boldsymbol{\eta})$ such that $\sigma\left(\mathcal{M}\left(\mathbf{z}, \boldsymbol{\eta}, t^{\star}(\mathbf{z}, \boldsymbol{\eta})\right)\right)=0$.

\subsection{Overview of DA normal form algorithm}

Given an origin-preserving Poincaré return map $\mathcal{M}$ of a repetitive Hamiltonian system where the components of the map are in phase space coordinates, the DANF algorithm (Berz 1999) provides a nonlinear change of variables by an order-by-order transformation to rotationally invariant normal form coordinates.

For parameter-dependent systems, the first step is determining the parameter-dependent fixed point $\delta \mathbf{z}_{\mathrm{FP}}(\delta \boldsymbol{\eta})$ of the origin-preserving map $\mathcal{M}(\delta \mathbf{z}, \delta \boldsymbol{\eta})$ such that $\mathcal{M}\left(\delta \mathbf{z}_{\mathrm{FP}}(\delta \boldsymbol{\eta}), \delta \boldsymbol{\eta}\right)=$ 
$\delta \mathbf{z}_{\mathrm{FP}}(\delta \boldsymbol{\eta})$. The parameter-dependent fixed point $\delta \mathbf{z}_{\mathrm{FP}}$ is determined by defining the extended map $\mathcal{N}=\left(\mathcal{M}-\mathcal{I}_{\delta \mathbf{z}}, \delta \boldsymbol{\eta}\right)$ and evaluating its inverse at the expansion point $\delta z=0$ :

$$
\left(\delta \mathbf{z}_{\mathrm{FP}}(\delta \boldsymbol{\eta}), \delta \boldsymbol{\eta}\right)=\mathcal{N}^{-1}(\mathbf{0}, \delta \boldsymbol{\eta}) .
$$

The map $\mathcal{M}$ is then expanded around its parameter-dependent fixed point $\delta \mathbf{z}_{\mathrm{FP}}$.

In the next step, the linear transformation, the system is diagonalized, transforming the map $\mathcal{M}$ into the complex conjugate eigenvector space of its linear part, assuming linearly stable behavior around the fixed point.

The following nonlinear transformations are determined separately in each of the complex conjugate eigenvector subspaces one order after another, starting with $m=2$. The transformations are given by $\mathcal{A}_{m}={ }_{m} \mathcal{I}+\mathcal{T}_{m}$, where $\mathcal{T}_{m}$ is a polynomial only of order $m$. Hence, the transformation $\mathcal{A}_{m}$ is a near-identity transformation and a full identify up to order $m-1$. The goal is finding $\mathcal{T}_{m}$ such that the $m$ th order of the map $\mathcal{M}_{m-1}$ is simplified or even eliminated when the transformation $\mathcal{A}_{m}$ and its inverse $\mathcal{A}_{m}^{-1}={ }_{m} \mathcal{I}-\mathcal{T}_{m}$ are applied to it. The following equations illustrate how $\mathcal{T}_{m}$ is determined.

Given the map $\mathcal{M}_{m-1}$, representing $\mathcal{M}$ simplified up to order $m-1$ and applying $\mathcal{A}_{m}$ and its inverse to it, yields Berz (1999, Eq. 7.60):

$$
\begin{aligned}
\mathcal{A}_{m} \circ \mathcal{M}_{m-1} \circ \mathcal{A}_{m}^{-1} & ={ }_{m}\left(\mathcal{I}+\mathcal{T}_{m}\right) \circ\left(\mathcal{R}+\mathcal{S}_{m}\right) \circ\left(\mathcal{I}-\mathcal{T}_{m}\right) \\
& ={ }_{m}\left(\mathcal{I}+\mathcal{T}_{m}\right) \circ\left(\mathcal{R}-\mathcal{R} \circ \mathcal{T}_{m}+\mathcal{S}_{m}\right) \\
& ={ }_{m} \mathcal{R}+\mathcal{S}_{m}+\left[\mathcal{T}_{m}, \mathcal{R}\right],
\end{aligned}
$$

where $\mathcal{R}$ is the diagonalized linear part and $\mathcal{S}_{m}$ represents only the $m$ th-order terms of the map $\mathcal{M}_{m-1}$ (the leading order of terms that are non-simplified yet). Note that the equations above only consider terms up to order $m$, since terms of order $m+1$ and larger are irrelevant for determining $\mathcal{T}_{m}$. The maximum simplification would be achieved by finding $\mathcal{T}_{m}$ such that the commutator $\mathcal{C}_{m}=\mathcal{T}_{m} \circ \mathcal{R}-\mathcal{R} \circ \mathcal{T}_{m}=\left[\mathcal{T}_{m}, \mathcal{R}\right]=-\mathcal{S}_{m}$, which would eliminate all terms of order $m$. This is not always possible, because for uneven orders the commutator has vanishing terms such that the associated terms in $\mathcal{S}_{m}$ cannot be canceled. The remaining terms of $\mathcal{S}_{m}$ describe the entire dynamics of the systems in a nutshell and are the key elements of the normal form and therefore essential for further dynamic analysis.

As a result of the order-by-order transformation, the map is significantly simplified up to an arbitrary order to a rotational invariant normal form map $\mathcal{M}_{\mathrm{NF}}$ yielding circular phase space behavior. The rotational invariance implies an interpretation of the normal form as an averaged representation of the original Poincaré return map $\mathcal{M}$, in the limit where the map application is repeated infinitely many times. The entire dynamics in the normal form is given by the angle advancement along the circular phase space curves, which is only dependent on the (normal form) radius $r_{\mathrm{NF}}$ of the circular curves.

Section 2.4 explains the key steps of the algorithm in detail for a one-dimensional system and is complemented by Weisskopf (2019) and its detailed step-by-step calculation of the DANF algorithm on a 1D example case. Specifics for higher-dimensional systems are also given in Weisskopf (2019) as well as Berz (1999), which also considers non-symplectic systems.

\subsection{Key steps in DANF algorithm}

Since the zonal bounded motion problem can be reduced to one-dimensional phase space motion (see Sect. 4), we will be considering the DANF algorithm in more detail for the 1D case. Weisskopf (2019) provides a very detailed calculation of the DANF algorithm for the 
one-dimensional phase space system of the Centrifugal Governor, which shall complement the following explanations.

The starting point for the DA normal form algorithm is an origin-preserving fixed-point phase space map $\mathcal{M}$, which expresses the final phase space state $\mathbf{z}_{f}=\left(Q_{0}, P_{0}\right)$ around a fixed point in terms of the initial phase space state $\mathbf{z}_{i}=\left(q_{0}, p_{0}\right)$ (around the same fixed point) within the same phase space.

To introduce our notation of the map and its coefficients, we will write the first few terms of the map $\mathcal{M}=\mathcal{L}+\sum_{m} \mathcal{U}_{m}$ explicitly

$$
\begin{aligned}
\mathcal{M}\left(q_{0}, p_{0}\right)= & \left(\begin{array}{l}
\mathcal{M}^{+}\left(q_{0}, p_{0}\right) \\
\mathcal{M}^{-}\left(q_{0}, p_{0}\right)
\end{array}\right)=\left(\begin{array}{l}
Q_{0}\left(q_{0}, p_{0}\right) \\
P_{0}\left(q_{0}, p_{0}\right)
\end{array}\right) \\
= & \underbrace{\left(\begin{array}{l}
\left(Q_{0} \mid q_{0}\right)\left(Q_{0} \mid p_{0}\right) \\
\left(P_{0} \mid q_{0}\right)
\end{array}\left(\begin{array}{l}
\left.P_{0} \mid p_{0}\right)
\end{array}\right)\left(\begin{array}{l}
q_{0} \\
p_{0}
\end{array}\right)\right.}_{\mathcal{L}} \\
& +\underbrace{\left(\begin{array}{l}
\mathcal{U}_{2(2,0)}^{+} \\
\mathcal{U}_{2(2,0)}^{-}
\end{array}\right) q_{0}^{2}+\left(\begin{array}{l}
\mathcal{U}_{2(1,1)}^{+} \\
\mathcal{U}_{2(1,1)}^{-}
\end{array}\right) q_{0} p_{0}+\left(\begin{array}{l}
\mathcal{U}_{2(0,2)}^{+} \\
\mathcal{U}_{2(0,2)}^{-}
\end{array}\right) p_{0}^{2}}_{\mathcal{U}_{2}} \\
& +\underbrace{\left(\begin{array}{l}
\mathcal{U}_{3(3,0)}^{+} \\
\mathcal{U}_{3(3,0)}^{-}
\end{array}\right) q_{0}^{3}+\left(\begin{array}{l}
\mathcal{U}_{3(2,1)}^{+} \\
\mathcal{U}_{3(2,1)}^{-}
\end{array}\right) q_{0}^{2} p_{0}+\cdots}_{\mathcal{U}_{3}},
\end{aligned}
$$

where $\mathcal{L}$ is the linear part and $\mathcal{U}_{m}$ denotes the nonlinear parts of order $m$. The position $Q$ and momentum $P$ components of the map correspond to the upper and lower components and are denoted with ' + ' and ' - ', respectively. The coefficients in the upper and lower $( \pm)$ components for the nonlinear $k(=a+b)$ th-order terms $x^{a} p^{b}$ are denoted with $\mathcal{U}_{k(a, b)}^{ \pm}$. The coefficients $(a \mid b)$ of the matrix $\hat{L}$ in the linear part $\mathcal{L}$ represent the factor with which $a$ is linearly dependent on $b$.

If the system is parameter dependent, the first step is determining the parameter-dependent fixed point $\delta \mathbf{z}_{\mathrm{FP}}$ as explained in Sect. 2.3 and expanding the map around it.

In the linear transformation of the DA normal form algorithm, the map is diagonalized, transforming it into the eigenvector space of the linear matrix $\hat{L}$. For this, we require that the transfer matrix $\hat{L}$ has distinct eigenvalues. Furthermore, we require that the system is linearly stable, which means that all of the distinct eigenvalues have an absolute value of $\leq 1$.

In this introduction, we are only considering the most common case: a symplectic system yielding a complex conjugate eigenvalue pair of magnitude one. The diagonal matrix $\hat{R}$ of the diagonalized linear part $\mathcal{R}$ is given by the complex conjugate eigenvalues on main diagonal

$$
\hat{R}=\left(\begin{array}{cc}
e^{i \mu} & 0 \\
0 & e^{-i \mu}
\end{array}\right)
$$

where $\mu$ is the complex phase of the eigenvalue. The linear transformation $\mathcal{A}_{1}$ and its inverse $\mathcal{A}_{1}^{-1}$ for the diagonalization have corresponding transformation matrices $\hat{A}_{1}$ and $\hat{A}_{1}^{-1}$, which are obtained in the usual fashion by using the complex conjugate eigenvectors of the linear matrix $\hat{L}$, such that $\hat{R}=\hat{A}_{1} \cdot \hat{L} \cdot \hat{A}_{1}^{-1}$. Since higher-order terms $\mathcal{U}_{m}$ are also transformed by the linear transformation, it is important that the determinant of the transformation and its inverse are of magnitude 1 (more details in Weisskopf (2019)). The result of the first step is the map $\mathcal{M}_{1}$ in the complex conjugate eigenvector basis of its linear part: 


$$
\mathcal{M}_{1}=\mathcal{A}_{1} \circ \mathcal{M} \circ \mathcal{A}_{1}^{-1}=\mathcal{A}_{1} \circ \mathcal{L} \circ \mathcal{A}_{1}^{-1}+\sum_{m} \mathcal{A}_{1} \circ \mathcal{U}_{m} \circ \mathcal{A}_{1}^{-1}=\mathcal{R}+\sum_{m} S_{m}
$$

where $\mathcal{S}_{m}$ are the transformed nonlinear parts of order $m$. Note that the upper and lower components of the map $\mathcal{M}_{1}$ are complex conjugate to each other just like the new variables $\left(q_{1}, p_{1}\right)$ of the map.

All the following nonlinear transformations are done one order after another, where the result of the $m$ th-order transformation is calculated via

$$
\mathcal{M}_{m}=\mathcal{A}_{m} \circ \mathcal{M}_{m-1} \circ \mathcal{A}_{m}^{-1} .
$$

The transformation $\mathcal{A}_{m}$ and its inverse $\mathcal{A}_{m}^{-1}$ are given by

$$
\mathcal{A}_{m}={ }_{m} \mathcal{I}+\mathcal{T}_{m} \quad \mathcal{A}_{m}^{-1}={ }_{m} \mathcal{I}-\mathcal{T}_{m},
$$

where $\mathcal{T}_{m}$ is a polynomial of only order $m$ terms (see Eq. 9 for example of $\mathcal{T}_{2}$ ).

The higher-order terms of the transformation $\mathcal{A}_{m}$ do not influence the $m$ th-order transformation and can therefore be chosen freely, i.e., to make the transformation symplectic (with $\mathcal{A}_{m}=\exp \left(L_{\mathcal{T}_{m}}\right)$ —see Weisskopf (2019)) or to avoid higher-order resonances between multiple dimensions. However, the higher orders of the resulting map $\mathcal{M}_{m}$ are strongly dependent on $\mathcal{A}_{m}$, its higher-order terms and its corresponding inverse. Weisskopf (2019) analyzes the influence of the second-order transformation on the third-order terms of the resulting map in great detail.

The second-order transformation has the following form

$$
\begin{aligned}
\mathcal{A}_{2} \circ \mathcal{M}_{1} \circ \mathcal{A}_{2}^{-1} & ={ }_{2}\left(\mathcal{I}+\mathcal{T}_{2}\right) \circ\left(\mathcal{R}+\mathcal{S}_{2}\right) \circ\left(\mathcal{I}-\mathcal{T}_{2}\right) \\
& ={ }_{2}\left(\mathcal{I}+\mathcal{T}_{2}\right) \circ\left(\mathcal{R}-\mathcal{R} \circ \mathcal{T}_{2}+\mathcal{S}_{2}\right) \\
& ={ }_{2} \mathcal{R}+\mathcal{S}_{2}+\left[\mathcal{T}_{2}, \mathcal{R}\right] .
\end{aligned}
$$

The goal is to find $\mathcal{T}_{2}$ such that $\left[\mathcal{T}_{2}, \mathcal{R}\right]=-\mathcal{S}_{2}$, where $\mathcal{T}_{2}$ is

$$
\begin{aligned}
\mathcal{T}_{2}(q, p) & =\left(\mathcal{T}_{2}^{ \pm} \mid 2,0\right) q^{2}+\left(\mathcal{T}_{2}^{ \pm} \mid 1,1\right) q p+\left(\mathcal{T}_{2}^{ \pm} \mid 0,2\right) p^{2} \\
& =\left(\begin{array}{c}
\mathcal{T}_{2(2,0)}^{+} \\
\mathcal{T}_{2(2,0)}^{-}
\end{array}\right) q^{2}+\left(\begin{array}{c}
\mathcal{T}_{2(1,1)}^{+} \\
\mathcal{T}_{2(1,1)}^{-}
\end{array}\right) q p+\left(\begin{array}{c}
\mathcal{T}_{2(0,2)}^{+} \\
\mathcal{T}_{2(0,2)}^{-}
\end{array}\right) p^{2} .
\end{aligned}
$$

The commutator $\mathcal{C}_{2}=\mathcal{T}_{2}, \mathcal{R}-\mathcal{R} \mathcal{T}_{2}=\left[\mathcal{T}_{2}, \mathcal{R}\right]$ from the second-order transformation is given by

$$
\begin{aligned}
{\left[\mathcal{T}_{2}, \mathcal{R}\right]=} & \left(\begin{array}{c}
\mathcal{T}_{2(2,0)}^{+}\left(e^{2 i \mu}-e^{i \mu}\right) \\
\mathcal{T}_{2(2,0)}^{-}\left(e^{2 i \mu}-e^{-i \mu}\right)
\end{array}\right) q^{2}+\left(\begin{array}{c}
-e^{i \mu} \mathcal{T}_{2(1,1)}^{+} \\
-e^{-i \mu} \mathcal{T}_{2(1,1)}^{-}
\end{array}\right) q p \\
& +\left(\begin{array}{c}
\mathcal{T}_{2(0,2)}^{+}\left(e^{-2 i \mu}-e^{i \mu}\right) \\
\mathcal{T}_{2(0,2)}^{-}\left(e^{-2 i \mu}-e^{-i \mu}\right)
\end{array}\right) p^{2} \\
= & \left(\mathcal{C}_{2}^{ \pm} \mid 2,0\right) q^{2}+\left(\mathcal{C}_{2}^{ \pm} \mid 1,1\right) q p+\left(\mathcal{C}_{2}^{ \pm} \mid 0,2\right) p^{2} .
\end{aligned}
$$

Note that none of the coefficients of $\mathcal{C}_{2}$ are zero, which means that the coefficients of $\mathcal{T}_{2}$ can be chosen such that the commutator $\mathcal{C}_{2}$ cancels the nonlinear terms of order two $\mathcal{S}_{2}$, so for $\left(\mathcal{C}_{2}^{ \pm} \mid k^{+}, k^{-}\right)=-\left(\mathcal{S}_{2}^{ \pm} \mid k^{+}, k^{-}\right)$we choose

$$
\left(\mathcal{T}_{2}^{ \pm} \mid k^{+}, k^{-}\right)=\frac{-\left(\mathcal{S}_{2}^{ \pm} \mid k^{+}, k^{-}\right)}{\left(e^{i \mu\left(k^{+}-k^{-}\right)}-e^{ \pm i \mu}\right)} .
$$


In the second-order transformation, all second-order terms of $\mathcal{M}_{1}$ will be canceled. Note that higher-order terms $\mathcal{S}_{m}$ with $m>2$ will change due to the second-order transformation and so will the complex conjugate variables from $\left(q_{1}, p_{1}\right)$ to $\left(q_{2}, p_{2}\right)$. The resulting map of the transformation will be $\mathcal{M}_{2}=\mathcal{R}+\sum_{k=3}^{n} \mathcal{S}_{k}$, where $\mathcal{S}_{k}$ of $\mathcal{M}_{2}$ are not equal to the $\mathcal{S}_{k}$ of $\mathcal{M}_{1}$.

The process for the third-order transformation is very similar to the second-order transformation with the exception that the commutator has at least one zero in the coefficients:

$$
\begin{aligned}
\mathcal{C}_{3}= & \left(\begin{array}{c}
\mathcal{T}_{3(3,0)}^{+}\left(e^{3 i \mu}-e^{i \mu}\right) \\
\mathcal{T}_{3(3,0)}^{-}\left(e^{3 i \mu}-e^{-i \mu}\right)
\end{array}\right) q^{3}+\left(\begin{array}{c}
0 \\
\left(e^{i \mu}-e^{-i \mu}\right) \mathcal{T}_{3(2,1)}^{-}
\end{array}\right) q^{2} p \\
& +\left(\begin{array}{c}
\left(e^{-i \mu}-e^{i \mu}\right) \mathcal{T}_{3(1,2)}^{-} \\
0
\end{array}\right) q p^{2}+\left(\begin{array}{c}
\mathcal{T}_{3(0,3)}^{+}\left(e^{-3 i \mu}-e^{i \mu}\right) \\
\mathcal{T}_{3(0,3)}^{-}\left(e^{-3 i \mu}-e^{-i \mu}\right)
\end{array}\right) p^{3} .
\end{aligned}
$$

The coefficients $\mathcal{C}_{3(2,1)}^{+}$and $\mathcal{C}_{3(1,2)}^{-}$are both zero, which means that the terms $\mathcal{S}_{3(2,1)}^{+}$and $\mathcal{S}_{3(1,2)}^{-}$cannot be eliminated. While we will not go there in detail, we want to note that generally only terms $\mathcal{S}_{k\left(\frac{k+1}{2}, \frac{k-1}{2}\right)}^{+}$and $\mathcal{S}_{k\left(\frac{k-1}{2}, \frac{k+1}{2}\right)}^{-}$for uneven orders $k$ survive the nonlinear transformations.

These surviving terms are the key structure of the normal form as the following steps will show. We will rewrite the map to make use of the special form of the surviving terms with

$$
\left(\begin{array}{l}
\mathcal{M}_{m}^{+} \\
\mathcal{M}_{m}^{-}
\end{array}\right)=\left(\begin{array}{c}
q_{m}\left(e^{+i \mu}+\sum_{k}\left(\mathcal{S}_{k}^{+} \mid k+1, k\right)\left(q_{m} p_{m}\right)^{k}\right) \\
p_{m}\left(e^{-i \mu}+\sum_{k}\left(\mathcal{S}_{k}^{-} \mid k, k+1\right)\left(q_{m} p_{m}\right)^{k}\right)
\end{array}\right)=\left(\begin{array}{c}
q_{m} f\left(q_{m} p_{m}\right) \\
p_{m} \bar{f}\left(q_{m} p_{m}\right)
\end{array}\right) .
$$

Furthermore, we are going to make use of the complex conjugate property of the upper and lower components and rewrite $f=e^{i \Lambda\left(q_{m} p_{m}\right)}$.

Since the original map $\mathcal{M}$ only operates in real space, the normal form map $\mathcal{M}_{\mathrm{NF}}$ should also only operate in real space. This is why the current map $\mathcal{M}_{m}$, where $m$ is the order of last transformation, is transformed to a real normal form basis $\left(q_{\mathrm{NF}}, p_{\mathrm{NF}}\right)$ composed of the real and imaginary parts of the current complex conjugate basis $\left(q_{m}, p_{m}\right)$ using the transformation $\mathcal{A}_{\text {real }}$ and its inverse (Berz 1999, eq 7.58,7.59 and 7.67):

$$
q_{\mathrm{NF}}=\left(q_{m}+p_{m}\right) / 2 \text { and } p_{\mathrm{NF}}=\left(q_{m}-p_{m}\right) / 2 i \text {. }
$$

In particular, this allows expressing $q_{m} p_{m}$ in terms of the squared normal form radius $r_{\mathrm{NF}}^{2}$ (Berz 1999, Eq. 7.67):

$$
q_{m} p_{m}=\left(q_{\mathrm{NF}}+i p_{\mathrm{NF}}\right)\left(q_{\mathrm{NF}}-i p_{\mathrm{NF}}\right)=\left(q_{\mathrm{NF}}\right)^{2}+\left(p_{\mathrm{NF}}\right)^{2}=r_{\mathrm{NF}}^{2} .
$$

The transformation into normal form coordinates $\left(q_{\mathrm{NF}}, p_{\mathrm{NF}}\right)$ is conducted as follows (Berz 1999, Eq. 7.68):

$$
\begin{aligned}
\mathcal{M}_{\mathrm{NF}}^{ \pm} & =\left(\begin{array}{c}
Q_{\mathrm{NF}}\left(q_{\mathrm{NF}}, p_{\mathrm{NF}}\right) \\
P_{\mathrm{NF}}\left(q_{\mathrm{NF}}, p_{\mathrm{NF}}\right)
\end{array}\right)=\left(\begin{array}{cc}
1 / 2 & 1 / 2 \\
1 / 2 i & -1 / 2 i
\end{array}\right) \cdot\left(q_{\mathrm{NF}} \pm i p_{\mathrm{NF}}\right) e^{ \pm i \Lambda\left(\left(q_{\mathrm{NF}}\right)^{2}+\left(p_{\mathrm{NF}}\right)^{2}\right)} \\
& =\left(\begin{array}{c}
q_{\mathrm{NF}}\left(e^{+i \Lambda\left(r_{\mathrm{NF}}^{2}\right)}+e^{-i \Lambda\left(r_{\mathrm{NF}}^{2}\right)}\right)+p_{\mathrm{NF}} i\left(e^{+i \Lambda\left(r_{\mathrm{NF}}^{2}\right)}-e^{-i \Lambda\left(r_{\mathrm{NF}}^{2}\right)}\right) \\
-q_{\mathrm{NF}} i\left(e^{+i \Lambda\left(r_{\mathrm{NF}}^{2}\right)}-e^{-i \Lambda\left(r_{\mathrm{NF}}^{2}\right)}\right)+p_{\mathrm{NF}}\left(e^{+i \Lambda\left(r_{\mathrm{NF}}^{2}\right)}+e^{-i \Lambda\left(r_{\mathrm{NF}}^{2}\right)}\right)
\end{array}\right) \\
& =\left(\begin{array}{c}
\cos \left(\Lambda\left(r_{\mathrm{NF}}^{2}\right)\right)-\sin \left(\Lambda\left(r_{\mathrm{NF}}^{2}\right)\right) \\
\sin \left(\Lambda\left(r_{\mathrm{NF}}^{2}\right)\right) \\
\cos \left(\Lambda\left(r_{\mathrm{NF}}^{2}\right)\right)
\end{array}\right) \cdot\left(\begin{array}{c}
q_{\mathrm{NF}} \\
p_{\mathrm{NF}}
\end{array}\right) .
\end{aligned}
$$

Equation 16 illustrates the circular phase space behavior in normal form coordinates with only amplitude $r_{\mathrm{NF}}$-dependent angle advancements $\Lambda$. 
The normalized constant part of $\Lambda$ is referred to as the tune $v$ in the beam physics terminology. The amplitude $r_{\mathrm{NF}}$-dependent changes are known as the tune shifts $\delta v$, so $v+\delta v\left(r_{\mathrm{NF}}^{2}\right)=\Lambda\left(r_{\mathrm{NF}}^{2}\right) / 2 \pi$.

The normal form transformation $\mathcal{A}$ and its inverse $\mathcal{A}^{-1}$ are given by

$$
\mathcal{M}_{\mathrm{NF}}=\underbrace{\mathcal{A}_{\text {real }} \circ \mathcal{A}_{m} \circ \mathcal{A}_{m-1} \circ \cdots \circ \mathcal{A}_{1}}_{\mathcal{A}} \circ \mathcal{M} \circ \underbrace{\mathcal{A}_{1}^{-1} \circ \cdots \circ \mathcal{A}_{m-1}^{-1} \circ \mathcal{A}_{m}^{-1} \circ \mathcal{A}_{\text {real }}^{-1}}_{\mathcal{A}^{-1}} .
$$

In particular, the normal form transformation $\mathcal{A}$ yields how the normal form variables $\left(q_{\mathrm{NF}}, p_{\mathrm{NF}}\right)$ depend on the original phase space variables $(q, p)$ and, if considered, system parameters $\boldsymbol{\eta}$ (see Weisskopf (2019)), which suggests the following notation for $\mathcal{A}$ and its inverse

$$
\begin{aligned}
\mathcal{A} & =\left(q_{\mathrm{NF}}(q, p, \boldsymbol{\eta}), p_{\mathrm{NF}}(q, p, \boldsymbol{\eta})\right), \\
\mathcal{A}^{-1} & =\left(q\left(q_{\mathrm{NF}}, p_{\mathrm{NF}}, \boldsymbol{\eta}\right), p\left(q_{\mathrm{NF}}, p_{\mathrm{NF}}, \boldsymbol{\eta}\right)\right) .
\end{aligned}
$$

For higher-dimensional cases, the general process does not change and can be considered in the complex conjugate eigenvector subspaces generated by the diagonalization. However, the commutator will include a resonance condition Berz (1999, Eq. 7.65), which might allow for the survival of more terms that would break the rotational invariance of the normal form [see Weisskopf (2019)].

\section{Zonal problem}

The zonal problem considers a gravitational field of an axially symmetric body. Accordingly, the system is described in cylindrical $(r, \phi, z)$-coordinates with the origin at the center of mass, $z$ along the symmetry axis and $\phi$ as the cyclic variable. The gravitational potential energy $U(r, z)$ is expressed by the zonal harmonics (Legendre Polynomials) $P_{l}$ and their corresponding zonal harmonic coefficients $J_{l}$ :

$$
U(r, z)=-\frac{\mu}{\rho}\left(1-\sum_{l=2}^{\infty} J_{l}\left(\frac{R_{0}}{\rho}\right)^{l} P_{l}\left(\frac{z}{\rho}\right)\right) \text { with } \rho=\sqrt{r^{2}+z^{2}} .
$$

In the case of the Earth, the $J_{2}$-term is the most dominate zonal perturbation representing the oblateness of the Earth. The following dimensionless units are used: Distances are considered in units of the Earth radius $R_{0}=6378.137 \mathrm{~km}$ and time is considered in units of $T_{0}=806.811 \mathrm{~s}$ such that the gravitational constant assumes the value $\mu=1$.

\subsection{Equations of motion}

Given the Lagrangian $L=\frac{1}{2}\left(\dot{r}^{2}+r^{2} \dot{\phi}^{2}+\dot{z}^{2}\right)-U(r, z)$ in cylindrical $(r, \phi, z)$-coordinates, the generalized velocities (canonical momenta) to the coordinates $r, z$ and $\phi$ are denoted with $v_{r}, v_{z}$ and $\mathcal{H}_{z}$, respectively, so that we have

$$
v_{r}=\frac{d L}{d \dot{r}}=\dot{r}, \quad v_{z}=\frac{d L}{d \dot{z}}=\dot{z}, \quad \text { and } \quad \mathcal{H}_{z}=\frac{d L}{d \dot{\phi}}=r^{2} \dot{\phi},
$$

where $\mathcal{H}_{z}=r^{2} \dot{\phi}$ is the angular momentum component along the symmetry axis and the canonical momentum to the angle $\phi$. The Lagrange-Euler equations show that $\mathcal{H}_{z}$ is a constant of motion, since $\frac{\partial L}{\partial \phi}=0=\mathrm{d}_{t} \mathcal{H}_{z}$. Using the Legendre transformation, the Hamiltonian 


$$
\begin{aligned}
H & =v_{r}^{2}+\frac{\mathcal{H}_{z}^{2}}{r^{2}}+v_{z}^{2}-\frac{v_{r}^{2}+v_{z}^{2}}{2}-\frac{\mathcal{H}_{z}^{2}}{2 r^{2}}+U(r, z) \\
& =\frac{v_{r}^{2}+v_{z}^{2}}{2}+\frac{\mathcal{H}_{z}^{2}}{2 r^{2}}+U(r, z)
\end{aligned}
$$

is obtained.

Due to the time independence of the system $\left(\mathrm{d}_{t} H=0\right)$, the Hamiltonian is equivalent to the energy

$$
E\left(r, v_{r}, v_{z}, \mathcal{H}_{z}\right)=\frac{v_{r}^{2}+v_{z}^{2}}{2}+\frac{\mathcal{H}_{z}^{2}}{2 r^{2}}+U(r, z)
$$

as the other constant of motion.

The equations of motion are derived from the Hamiltonian via the Hamilton equations

$$
\begin{aligned}
\dot{r} & =v_{r}, \quad \dot{z}=v_{z}, \quad \dot{\phi}=\frac{\mathcal{H}_{z}}{r^{2}}, \\
\dot{v}_{r} & =\frac{\mathcal{H}_{z}^{2}}{r^{3}}-\frac{\partial U}{\partial r}, \quad \dot{v}_{z}=-\frac{\partial U}{\partial z} \quad \text { and } \quad \dot{\mathcal{H}}_{z}=0,
\end{aligned}
$$

which show that the dynamics of the system are only dependent on the reduced state $\mathcal{Z}=$ $\left(r, v_{r}, z, v_{z}\right)^{T}$.

The time evolution $\mathcal{X}(t)$ of the state $\mathcal{X}=\left(r, v_{r}, z, v_{z}, \phi\right)^{T}$ of a spacecraft is determined by the integration of the system of ODE's $\dot{\mathcal{X}}=f\left(\mathcal{X}, \mathcal{H}_{z}\right)$, where the components of $f\left(\mathcal{X}, \mathcal{H}_{z}\right)$ are given by the right-hand side of the equations of motion (Eqs. 25 and 26). The orbit $\mathcal{O}$ of the spacecraft is described by the set of all states $\mathcal{X}(t)$.

\subsection{Bounded motion}

In the unperturbed case, with a spherically symmetric potential, every orbit is closed and forms a fixed elliptic shape in space. In particular, every orbit intersects with the equatorial plane in two places (excluding orbits of zero inclination). The intersection from south to north is called the ascending node $\Omega$. Due to zonal perturbation, the orbits do not close (Cook 1962), since the ascending node moves with every revolution around the Earth. The angular difference between two consecutive ascending nodes is denoted with $\Delta \Omega$, the drift of the right ascension of the ascending node $(\Omega)$. It is defined by

$$
\Delta \Omega=\phi\left(\Omega_{n+1}\right)-\phi\left(\Omega_{n}\right)-2 \pi \operatorname{sgn}\left(\mathcal{H}_{z}\right),
$$

where $-2 \pi \operatorname{sgn}\left(\mathcal{H}_{z}\right)$ ensures that $\Delta \Omega$ is the shortest angular distance between the two consecutive ascending nodes. The time between two consecutive ascending nodes is denoted with the nodal period $T_{d}=t\left(\Omega_{n+1}\right)-t\left(\Omega_{n}\right)$. The nodal period and the $\Omega$-drift show oscillatory behavior over multiple revolutions, which are periodic with a frequency $\omega_{p}$ corresponding to rotation frequency of the orbit/its apsides within its respective orbital plane. The oscillatory behavior of $T_{d}$ and $\Delta \Omega$ is illustrated in Fig. 2. Quasi-circular orbits show periodic behavior in the reduced dynamics of Eqs. 25 and 26, and the amplitude of the oscillation of $T_{\mathrm{d}}$ and $\Delta \Omega$ is zero for those orbits.

$\mathrm{Xu}$ et al. (2012) showed that the conditions for bounded motion between two orbits $\mathcal{O}_{1}$ and $\mathcal{O}_{2}$ in the zonal problem require the following conditions to be met:

$$
\begin{aligned}
\bar{T}_{d}\left(\mathcal{O}_{1}\right) & =\bar{T}_{d}\left(\mathcal{O}_{2}\right), \\
\overline{\Delta \Omega}\left(\mathcal{O}_{1}\right) & =\overline{\Delta \Omega}\left(\mathcal{O}_{2}\right) .
\end{aligned}
$$


In other words, any two orbits are in sync, if their average nodal period $\bar{T}_{d}$ and average drift of the ascending node $\overline{\Delta \Omega}$ are the same.

\section{Poincaré return map calculation and averaging}

The goal is to generate a Poincaré return map $\mathcal{P}$ that describes the dynamics of the system by characterizing how a state $\left(\mathcal{X}_{i n i}, t=0\right)$ within a Poincaré surface $\mathbb{S}$ belonging to an orbit $\mathcal{O}$ returns to/is mapped back onto $\mathbb{S}$ after each revolution of the orbit $\left(\mathcal{X}_{\text {fin }}, T_{\mathrm{d}}\right)$. Defining a suitable Poincare surface is the first step in generating the map. Secondly, a reference orbit with fixed-point properties has to be identified to ensure that the expansion point of the map returns to itself after each revolution. The Poincaré return map is then calculated as an expansion around the reference orbit before being averaged using DA normal form methods. This yields the average nodal period $\bar{T}_{\mathrm{d}}$ and average ascending node drift $\overline{\Delta \Omega}$ as a function of the system parameters and expansion variables around the reference orbit. Using DA inversion methods, the system parameters can be determined such that the bounded motion conditions are met.

\subsection{Poincaré surface space}

The subsection on bounded motion (Sect. 3.2) indicated the importance of the ascending node for various definitions in the zonal problem, which is why the Poincare surface is chosen to be equivalent to the set of all ascending nodes $\Omega$. The Poincaré surface $\mathbb{S}_{\Omega}$ can be divided into subsurfaces $\mathbb{S}_{\Omega, \mathcal{H}_{z}, E}$ for specific angular momentum components $\mathcal{H}_{z}$ and energies $E$. These surfaces contain all states with the parameters $\left(\mathcal{H}_{z}, E\right)$ that lie in the equatorial plane $(z=0)$ and satisfy $v_{z}>0$. The restriction of $v_{z}$ to positive values makes the relation between $E$ and $v_{z}$ (Eq. 24) bijective and therefore locally invertible in $\mathbb{S}_{\Omega, \mathcal{H}_{z}, E}$, so

$$
\mathbb{S}_{\Omega, \mathcal{H}_{z}, E}=\left\{\mathcal{X} \mid z=0, v_{z}=\sqrt{2(E-U(r))-v_{r}^{2}-\left(\frac{\mathcal{H}_{z}}{r}\right)^{2}}\right\} .
$$

This means that any state $\mathcal{X} \in \mathbb{S}_{\Omega_{,}, \mathcal{H}_{z}, E}$ is uniquely determined by $\left(r, v_{r}, \phi\right)$, since $z=0$ and $v_{z}\left(r, v_{r}, \mathcal{H}_{z}, E\right)$.

\subsection{Fixed-point orbits}

The orbit associated with the fixed-point state is called reference orbit. The reference orbit has the special property that it returns to the same reduced state $\mathcal{Z}=\left(r, v_{r}, z, v_{z}\right)^{T}$ after each revolution with a constant nodal period $T_{d}^{\star}$ and a constant angle advancement in $\phi$, which is also referred to as the fixed-point drift in the ascending node $\Delta \Omega^{\star}$.

For a certain set of parameters $\left(\mathcal{H}_{z}, E\right)$, we use DA inversion techniques iteratively to find the fixed-point orbit. The iteration is initialized with the state

$$
\mathcal{Z}_{0}=\left(r=-1 /(2 E), \quad v_{r}=0, \quad z=0, \quad v_{z}\left(r, \mathcal{H}_{z}, E\right)\right)^{T}
$$

at its ascending node $\Omega\left(v_{z}>0\right)$ and the state is expanded in the variables $\left(r, v_{r}\right)$. After a full orbit integration until the next ascending node intersection, the map $\mathcal{M}$ is timewise projected onto the Poincaré surface $\mathbb{S}_{\Omega, \mathcal{H}_{z}, E}$ (Sect. 2.2). The resulting Poincaré map $\mathcal{P}$ represents a one turn map in dependence on variations $\left(\delta r, \delta v_{r}\right)$ in the variables $\left(r, v_{r}\right)$. The difference 
between the constant part of the map $\mathcal{P}$ and the initial state $\mathcal{Z}_{0}$ in the components $r$ and $v_{r}$ is denoted with $\Delta r$ and $\Delta v_{r}$, respectively. The Poincaré map without its constant part is indicated by $\mathcal{P}^{\prime}$. The next initial state $\mathcal{Z}_{1}$ for the iterative process will be given by the evaluation of

$$
\left(\begin{array}{c}
\mathcal{Z}_{r, 1} \\
\mathcal{Z}_{v_{r}, 1}
\end{array}\right)=\left(\begin{array}{c}
\mathcal{P}_{r}^{\prime}\left(\delta r, \delta v_{r}\right)-\delta r \\
\mathcal{P}_{v_{r}}^{\prime}\left(\delta r, \delta v_{r}\right)-\delta v_{r}
\end{array}\right)^{-1}\left(\delta r=-\Delta r, \delta v_{r}=-\Delta v_{r}\right) .
$$

The process is repeated until the offset $\left(\Delta r, \Delta v_{r}\right)$ is smaller than a threshold value, i.e., $1 \mathrm{E}-14$.

\subsection{Calculation of Poincaré return map}

Given a fixed-point state $\mathcal{Z}^{\star}$ from Sect. 4.2 for the parameter set $\left(\mathcal{H}_{z}, E\right)$, the Poincaré return map $\mathcal{P}:\left(\mathbb{S}_{\Omega}, t\right) \rightarrow\left(\mathbb{S}_{\Omega}, t\right)$ is calculated as a DA expansion around that reference orbit. In the first step, the flow $\mathcal{M}$ of the fixed point and its neighborhood in $\mathbb{S}_{\Omega}$ (expansion in $\left.\left(\delta r, \delta v_{r}, \delta \mathcal{H}_{z}, \delta E\right)\right)$ is obtained by integrating the system of ODE's from the initial state until the reference/fixed-point orbit is an element of $\mathbb{S}_{\Omega, \mathcal{H}_{z}, E}$ again after $T_{\mathrm{d}}^{\star}$. In other words, the state is integrated until the orbit of $\mathcal{X}^{0}$ intersects with the equatorial plane from south to north again.

While the reference orbit itself is in $\mathbb{S}_{\Omega, \mathcal{H}_{z}, E} \subset \mathbb{S}_{\Omega}$ after $T_{\mathrm{d}}^{\star}$, the expansion around the reference orbit is not in $\mathbb{S}_{\delta, \mathcal{H}_{z}+\delta \mathcal{H}_{z}, E+\delta E} \subset \mathbb{S}_{\delta}$ due to changing nodal periods of the orbits within the expansion. In order to project the flow $\mathcal{M}$ after $T_{\mathrm{d}}^{\star}$ onto the Poincaré surface $\mathbb{S}_{\Omega, E+\delta \mathcal{H}_{z}, E+\delta \mathcal{H}_{z}}$, a timewise projection is calculated following Sect. 2.2 and Grote et al. (2006). The flow $\mathcal{M}$ is expanded in time to find the intersection time $t_{\text {intersec }}\left(\delta r, \delta v_{r}, \delta \mathcal{H}_{z}, \delta E\right)$ such that

$$
\mathcal{P}_{z}=\mathcal{M}_{z}\left(\delta r, \delta v_{r}, \delta \mathcal{H}_{z}, \delta E, t_{\text {intersec }}\left(\delta r, \delta v_{r}, \delta \mathcal{H}_{z}, \delta E\right)\right)=0
$$

and $\mathcal{P}=\left(\mathcal{M}\left(t_{\text {intersec }}\right), T_{\mathrm{d}}^{\star}+t_{\text {intersec }}\right) \in\left(\mathbb{S}_{\Omega, \mathcal{H}_{z}+\delta \mathcal{H}_{z}, E+\delta E}, t\right) \subset\left(\mathbb{S}_{\Omega}, t\right)$.

The time component $\mathcal{P}_{T_{\mathrm{d}}}$ of the Poincaré return map yields the dependence of the nodal period $T_{\mathrm{d}}$ on the system parameters and expansion variables.

\subsection{Normal form averaging}

Given the fixed-point Poincaré return map $\mathcal{P}$ from Sect. 4.3 with

$$
\mathcal{P}\left(\delta r, \delta v_{r}, \delta \mathcal{H}_{z}, \delta E\right)=\left(\begin{array}{c}
\mathcal{P}_{r}\left(\delta r, \delta v_{r}, \delta \mathcal{H}_{z}, \delta E\right) \\
\mathcal{P}_{v_{r}}\left(\delta r, \delta v_{r}, \delta \mathcal{H}_{z}, \delta E\right) \\
\mathcal{P}_{z}=0 \\
\mathcal{P}_{v_{z}}\left(\delta r, \delta v_{r}, \delta \mathcal{H}_{z}, \delta E\right) \\
\mathcal{P}_{\phi}\left(\delta r, \delta v_{r}, \delta \mathcal{H}_{z}, \delta E\right) \\
\mathcal{P}_{T_{d}}\left(\delta r, \delta v_{r}, \delta \mathcal{H}_{z}, \delta E\right)
\end{array}\right),
$$

we are using only the first two components (in $r$ and $v_{r}$ ) of the Poincare map for the calculation of phase space transformation provided by the DA normal form algorithm, since the motion is determined by only the $\left(r, v_{r}\right)$ phase space and the parameters $\left(\mathcal{H}_{z}, E\right)$. The reduced map is denoted with $\mathcal{K}=\left(\mathcal{P}_{r}, \mathcal{P}_{v_{r}}\right)^{T}$.

The DA normal form algorithm yields the normal form transformation $\mathcal{A}\left(\delta r, \delta v_{r}, \delta \mathcal{H}_{z}, \delta E\right)$ (see Eq. 18) such that

$$
\mathcal{A} \circ \mathcal{K} \circ \mathcal{A}^{-1}\left(q_{\mathrm{NF}}, p_{\mathrm{NF}}, \delta \mathcal{H}_{z}, \delta E\right)=\mathcal{K}_{\mathrm{NF}}\left(q_{\mathrm{NF}}, p_{\mathrm{NF}}, \delta \mathcal{H}_{z}, \delta E\right)
$$



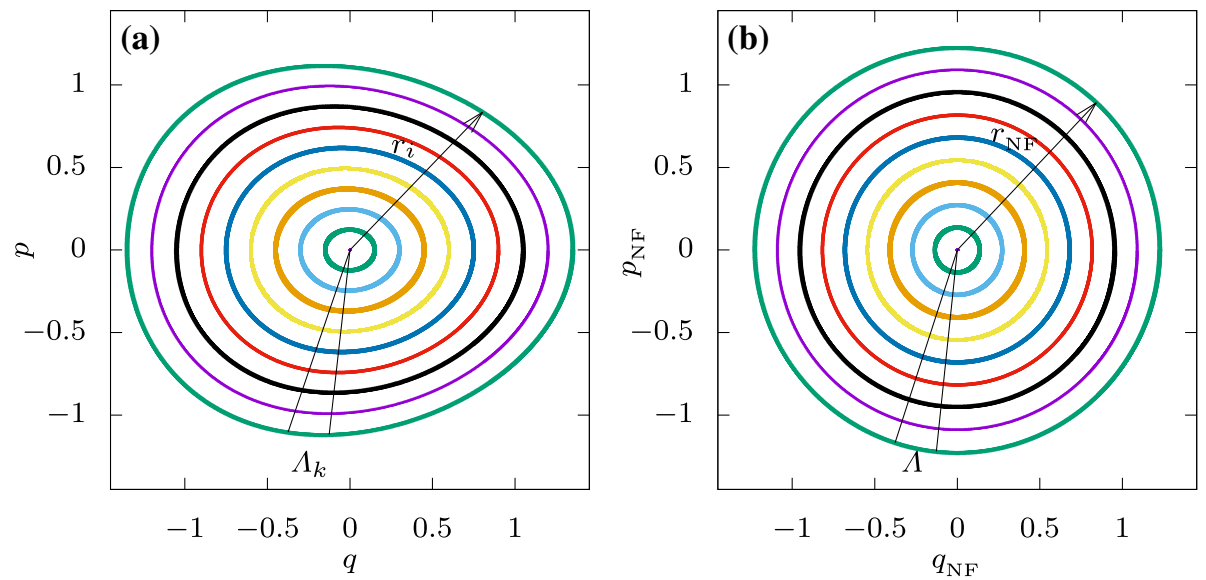

Fig. 1 a Non-circular behavior in the original phase space $(q, p)$ and $\mathbf{b}$ circular behavior in the corresponding normal form phase space $\left(q_{\mathrm{NF}}, p_{\mathrm{NF}}\right)$. In a, the phase space angle advancement $\Lambda_{k}$ and the phase space radius $r_{i}$ are not constant by continuously change along each of the phase space curves. In $\mathbf{b}$, the phase space behavior is rotationally invariant ('normalized') with a constant normal form radius $r_{\mathrm{NF}}$ and a constant, but amplitude-dependent, angle advancement $\Lambda\left(r_{\mathrm{NF}}\right)$

is rotational invariant in the normal form phase space coordinates $\left(q_{\mathrm{NF}}, p_{\mathrm{NF}}\right)$ up to the order of calculation. In other words, the phase space curves in $\left(\mathcal{P}_{r}\left(\delta r, \delta v_{r}, \delta \mathcal{H}_{z}, \delta E\right), \mathcal{P}_{v_{r}}\left(\delta r, \delta v_{r}\right.\right.$, $\left.\left.\delta \mathcal{H}_{z}, \delta E\right)\right)$ are transformed to circles in the normal form $\left(Q_{\mathrm{NF}}\left(q_{\mathrm{NF}}, p_{\mathrm{NF}}, \delta \mathcal{H}_{z}, \delta E\right), P_{\mathrm{NF}}\left(q_{\mathrm{NF}}\right.\right.$, $\left.\left.p_{\mathrm{NF}}, \delta \mathcal{H}_{z}, \delta E\right)\right)$ phase space as illustrated in Fig. 1.

By rewriting the normal form coordinates $\left(q_{\mathrm{NF}}, p_{\mathrm{NF}}\right)$ in an action-angle representation $\left(r_{\mathrm{NF}}, \Lambda\right)$ with

$$
\left(\begin{array}{c}
q_{\mathrm{NF}} \\
p_{\mathrm{NF}}
\end{array}\right)=r_{\mathrm{NF}}\left(\begin{array}{c}
\cos \Lambda \\
\sin \Lambda
\end{array}\right)
$$

each normal form phase space curve is characterized by the normal form radius (action) $r_{\mathrm{NF}}$ and the path along each curve is parameterized by the angle $\Lambda$. Using the inverse normal form transformation $\mathcal{A}^{-1}$ (see Eq. 19), the original phase space variables $\left(\delta r, \delta v_{r}\right)$ of $\mathcal{P}$ (and $\mathcal{K})$ are expressed in terms of the action-angle representation and variations in the system parameters $\left(\delta \mathcal{H}_{z}, \delta E\right)$ :

$$
\left(\delta r, \delta v_{r}\right)=\mathcal{A}^{-1}\left(q_{\mathrm{NF}}\left(r_{\mathrm{NF}}, \Lambda\right), p_{\mathrm{NF}}\left(r_{\mathrm{NF}}, \Lambda\right), \delta \mathcal{H}_{z}, \delta E\right) .
$$

The Poincaré map $\mathcal{P}\left(r_{\mathrm{NF}}, \Lambda, \delta \mathcal{H}_{z}, \delta E\right)$ is then averaged over a full phase space revolution, by integrating along the angle $\Lambda$ :

$$
\overline{\mathcal{P}}\left(r_{\mathrm{NF}}, \delta \mathcal{H}_{z}, \delta E\right)=\frac{1}{2 \pi} \oint \mathcal{P}\left(r_{\mathrm{NF}}, \Lambda, \delta \mathcal{H}_{z}, \delta E\right) d \Lambda .
$$

The numerical averaging presented in He et al. (2018) is done in the time domain, which cannot incorporate the slightly different oscillation frequencies of the relevant quantities $T_{\mathrm{d}}$ and $\Delta \Omega$ for the different orbits. The key advantage of the normal form representation is that the different oscillation frequencies are captured by the amplitude-dependent angle advancement in the normal form. The generalized parameterization of all normal form phase space curves makes the averaging independent of those differences in the frequency. 
Splitting the integration into subsections minimizes the error of the numerical integration and considerably improves the quality and accuracy of the averaging. For $n$ separate parameterization

$$
\left(\begin{array}{l}
q_{\mathrm{NF}} \\
q_{\mathrm{NF}}
\end{array}\right)=r_{\mathrm{NF}}\left(\begin{array}{cc}
\cos \left(\frac{2 \pi(k-1)}{n}\right) & -\sin \left(\frac{2 \pi(k-1)}{n}\right) \\
\sin \left(\frac{2 \pi(k-1)}{n}\right) & \cos \left(\frac{2 \pi(k-1)}{n}\right)
\end{array}\right)\left(\begin{array}{c}
\cos \Lambda \\
\sin \Lambda
\end{array}\right) k \in\{1,2, \ldots, n\},
$$

each section is integrated over the symmetric interval of $\Lambda \in\left[-\frac{\pi}{n}, \frac{\pi}{n}\right]$.

The result of the averaging yields every component of $\mathcal{P}$ averaged over a full phase space curve. In particular, it will yield the averaged drift in the ascending node $\overline{\Delta \Omega}\left(r_{\mathrm{NF}}, \delta \mathcal{H}_{z}, \delta E\right)$ and average nodal period $\bar{T}_{d}\left(r_{\mathrm{NF}}, \delta \mathcal{H}_{z}, \delta E\right)$.

For mission design purposes, the abstract quantity $r_{\mathrm{NF}}$ is expressed by the original coordinates $\left(\delta r, \delta v_{r}\right)$ and the parameters $\left(\delta \mathcal{H}_{z}, \delta E\right)$ with

$$
r_{\mathrm{NF}}^{2}\left(\delta r, \delta v_{r}, \delta \mathcal{H}_{z}, \delta E\right)=\left(q_{\mathrm{NF}}^{2}+p_{\mathrm{NF}}^{2}\right)\left(\delta r, \delta v_{r}, \delta \mathcal{H}_{z}, \delta E\right)
$$

using the normal form transformation $\mathcal{A}$, which yields how $\left(q_{\mathrm{NF}}, p_{\mathrm{NF}}\right)$ depend on the original coordinates $\left(\delta r, \delta v_{r}\right)$ and the parameters $\left(\delta \mathcal{H}_{z}, \delta E\right)$.

The average drift in the ascending node $\overline{\Delta \Omega}\left(\delta r, \delta v_{r}, \delta \mathcal{H}_{z}, \delta E\right)$ and the average nodal period $\bar{T}_{d}\left(\delta r, \delta v_{r}, \delta \mathcal{H}_{z}, \delta E\right)$ are then projected such that the bounded motion conditions are satisfied, with

$$
\begin{aligned}
\Delta \Omega^{\star} & =\overline{\Delta \Omega}\left(\delta r, \delta v_{r}, \delta \mathcal{H}_{z}\left(\delta r, \delta v_{r}\right), \quad \delta E\left(\delta r, \delta v_{r}\right)\right), \\
T_{d}^{\star} & =\bar{T}_{d}\left(\delta r, \delta v_{r}, \delta \mathcal{H}_{z}\left(\delta r, \delta v_{r}\right), \quad \delta E\left(\delta r, \delta v_{r}\right)\right) .
\end{aligned}
$$

In this process, DA inversion methods are used to find $\delta \mathcal{H}_{z}\left(\delta r, \delta v_{r}\right)$ and $\delta E\left(\delta r, \delta v_{r}\right)$. The dependence of $\mathcal{H}_{z}$ and $E$ on orbital parameters for bounded motion orbits became apparent already in Vadali et al. (1999) and Schaub and Alfriend (2001).

Theoretically, one could have proceeded with the abstract invariant of motion $r_{\mathrm{NF}}$ to satisfy the bounded motion condition with $\delta \mathcal{H}_{z}\left(r_{\mathrm{NF}}\right)$ and $\delta E\left(r_{\mathrm{NF}}\right)$. For specific bounded orbits, one would then have chosen a value for $r_{\mathrm{NF}}$ to calculate $\left(\delta \mathcal{H}_{z}, \delta E\right)$ and afterward the initial values for $\left(r, v_{r}\right)$ by using Eq. 37, where $\Lambda$ can be chosen freely.

\section{Results}

In the following section, we will apply the normal form methods for bounded motion of low Earth and medium Earth orbits. For this, we use fixed-point orbits of the zonal problem that have previously been investigated by He et al. (2018) for the low Earth orbit and Baresi and Scheeres (2017b) for the medium Earth orbit. As explained above, the fixed-point Poincaré maps $\mathcal{P}$ are calculated as an expansion in the variables $\left(\delta r, \delta v_{r}, \delta \mathcal{H}_{z}, \delta E\right)$ around the respective fixed-point orbit. In the calculation, we consider zonal perturbations up to the $J_{15}$-term (list of the used values of the coefficients $J_{2}$ to $J_{15}$ in "Appendix" Table 8), since investigations in He et al. (2018) indicated only little influence of $J_{k}$ terms for $k>15$. We are using maps of eighth order, which provide the best balance of accuracy and computation time.

It will be shown that the DANF method provides entire sets of bounded motions that extend far beyond the realistic/practical scope. Since the approach is based on polynomial expansions, it is obvious that it will have to fail at some point. In the last part of this section, 
Table 1 The expansion of $\mathcal{H}_{z}\left(\delta r, \delta v_{r}=0\right)$ and $E\left(\delta r, \delta v_{r}=0\right)$ for relative bounded motion orbits with an average nodal period

$\overline{T_{d}}=7.64916169(\approx 103 \mathrm{~min})$ and an average ascending node drift of $\overline{\Delta \Omega}=1.22871195 \mathrm{E}-3$ $\mathrm{rad}$. The expansion is relative to the pseudo-circular LEO from $\mathrm{He}$ et al. (2018)

\begin{tabular}{llll}
\hline $\mathcal{H}_{z}\left(\delta r, \delta v_{r}=0\right)=$ & & $E\left(\delta r, \delta v_{r}=0\right)=$ \\
\hline-0.16707295 & & -0.43870527 & \\
+0.32072807 & $\delta r^{2}$ & $-0.31602983 \mathrm{E}-3$ & $\delta r^{2}$ \\
$+0.25767948 \mathrm{E}-3$ & $\delta r^{3}$ & $-0.25390482 \mathrm{E}-6$ & $\delta r^{3}$ \\
-0.19132824 & $\delta r^{4}$ & $-0.31003174 \mathrm{E}-3$ & $\delta r^{4}$ \\
$+0.53296708 \mathrm{E}-4$ & $\delta r^{5}$ & $-0.85361819 \mathrm{E}-6$ & $\delta r^{5}$ \\
$+0.12006391 \mathrm{E}-1$ & $\delta r^{6}$ & $-0.32152252 \mathrm{E}-3$ & $\delta r^{6}$ \\
$+0.60713391 \mathrm{E}-3$ & $\delta r^{7}$ & $-0.24661573 \mathrm{E}-5$ & $\delta r^{7}$ \\
-0.19751494 & $\delta r^{8}$ & $-0.21784073 \mathrm{E}-3$ & $\delta r^{8}$ \\
\hline
\end{tabular}

we take a look at the limitations of the DANF method and the resulting sets for very large distances between orbits.

\subsection{Bounded motion in low Earth orbit}

In a first comparison, we are investigating bounded motion around a pseudo-circular low Earth orbit (LEO) that was also considered in He et al. (2018). The pseudo-circular orbit corresponds to the reduced fixed-point state

$$
\left(r^{\star}, v_{r}^{\star}\right)=(1.14016749,-1.05621369 \mathrm{E}-3)
$$

for the parameters $\left(\mathcal{H}_{z}, E\right)=(-0.16707295,-0.43870527)$. The orbit has a fixed nodal period of $T_{d}^{\star}=7.64916169(\approx 103 \mathrm{~min})$ and a constant ascending node drift of $\Delta \Omega^{\star}=$ $1.22871195 \mathrm{E}-3 \mathrm{rad}\left(0.0704^{\circ}\right)$. The $z$-phase space components of the Poincaré fixed-point orbit are defined by the Poincaré section $(z=0)$ and Eq. 30 with $v_{z}^{\star}\left(r^{\star}, v_{r}^{\star}, \mathcal{H}_{z}, E\right)=$ 0.92518953 .

The computation of the Poincaré map took 165 s on a Lenovo E470 with an Intel@Core ${ }^{T M}$ i5-7200U CPU $2.5 \mathrm{GHz}$. The map confirms the fixed-point property of the orbit, since the offset of the constant part of the map from the initial coordinates is well within the numerical error of the integration with $\left(\Delta r, \Delta v_{r}, \Delta z, \Delta v_{z}\right)=(4 \mathrm{E}-15,5 \mathrm{E}-13,-1 \mathrm{E}-15,-4 \mathrm{E}-15)$. The normal form transformation of the reduced fixed-point Poincaré map $\mathcal{K}=\left(\mathcal{P}_{r}, \mathcal{P}_{v_{r}}\right)^{T}$ is calculated via the DA normal form algorithm (in $90 \mathrm{~ms}$ ). The circular phase space behavior in normal form space is parameterized using the action-angle notation $\left(r_{\mathrm{NF}}, \Lambda\right)$. The phase space parameterization is then transformed back to the original coordinates of the Poincaré map. The Poincaré map is then averaged (in $52 \mathrm{~ms}$ ) over a full phase space rotation using eight subsections following the procedure outlined in Sect. 4.4. Afterward, the variable $r_{\mathrm{NF}}$ is expressed in terms of $\delta r, \delta v_{r}, \delta \mathcal{H}_{z}$ and $\delta E$ before the variations in the constants of motion $\left(\delta \mathcal{H}_{z}, \delta E\right)$ are matched dependent on $\left(\delta r, \delta v_{r}\right)$ such that the averaged expressions for $T_{d}$ and $\Delta \Omega$ satisfy the bounded motion conditions (Eqs. 41 and 42 ).

The dependence of the constants of motion $\left(\mathcal{H}_{z}, E\right)$ on $\left(\delta r, \delta v_{r}\right)$ for bounded motion around the pseudo-circular LEO from He et al. (2018) is given in Table 9 in "Appendix." Considering bounded orbits initiated with the same $v_{r}$ as the pseudo-circular orbit $\left(\delta v_{r}=0\right)$, the dependence of $\mathcal{H}_{z}\left(\delta r, \delta v_{r}=0\right)$ and $E\left(\delta r, \delta v_{r}=0\right)$ is provided in Table 1.

To show that the expansion of $\delta \mathcal{H}_{z}$ and $\delta E$ provides relative bounded motion orbits, we illustrate the long-term behavior of three LEOs relative to one another. The first orbit is the fixed-point/pseudo-circular orbit and is denoted with $\mathcal{O}_{0}$. The other two orbits are initiated at 
Table 2 All LEOs are initiated with $v_{r, 0}=-1.05621369 \mathrm{E}-3$ and $r_{0}=1.14016749+\delta r$ and have an average nodal period of $\overline{T_{d}}=7.64916169(\approx 103 \mathrm{~min})$ and an average ascending node drift of $\overline{\Delta \Omega}=1.22871195 \mathrm{E}-3$ rad. $\mathcal{O}_{0}$ is the pseudo-circular LEO from He et al. (2018)

\begin{tabular}{lllllr}
\hline & $\delta r$ & $\delta v_{r}$ & $\phi$ & $\mathcal{H}_{z}$ & \multicolumn{1}{c}{$E$} \\
\hline $\mathcal{O}_{0}$ & 0.00 & 0 & $0.0^{\circ}$ & -0.16707295 & -0.43870527 \\
$\mathcal{O}_{1}$ & $0.06(383 \mathrm{~km})$ & 0 & $0.5^{\circ}$ & -0.16592075 & -0.43870642 \\
$\mathcal{O}_{2}$ & $0.13(829 \mathrm{~km})$ & 0 & $0.5^{\circ}$ & -0.16170668 & -0.43871071 \\
\hline
\end{tabular}

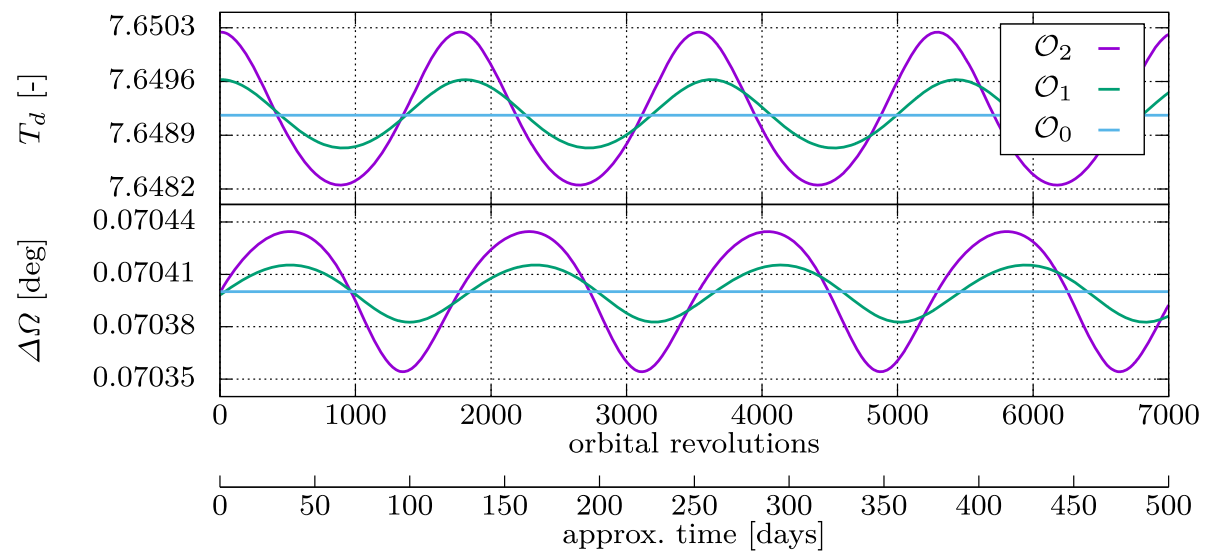

Fig. 2 Oscillatory behavior of the bounded motion quantities $T_{\mathrm{d}}$ and $\Delta \Omega$ of the bounded LEOs $\mathcal{O}_{1}$ and $\mathcal{O}_{2}$ initiated at $\delta r=0.06$ and $\delta r=0.13$, respectively. Additionally, the constant nodal period $T_{d}^{\star}=7.64916169$ and constant ascending node drift of $\Delta \Omega^{\star}=0.0704^{\circ}$ of the fixed-point orbit $\mathcal{O}_{0}$ are shown. The periods of oscillation are 1763 orbital revolutions (126 days) for $\mathcal{O}_{2}, 1810$ orbital revolutions (129 days) for $\mathcal{O}_{1}$ and 1823 orbital revolutions (130 days) for $\delta r \rightarrow 0$ of $\mathcal{O}_{0}$. The shown results are generated by numerical integration. The time domain was added assuming that on average one orbital revolution $\hat{\approx} T_{\mathrm{d}}^{\star}$

$\delta r=0.06$ with $\delta v_{r}=0\left(\mathcal{O}_{1}\right)$ and $\delta r=0.13$ with $\delta v_{r}=0\left(\mathcal{O}_{2}\right)$, respectively, and both have an initial longitudinal offset of $\phi=0.5^{\circ}$ relative to $\mathcal{O}_{0}$. The specific values of the orbits are given in Table 2.

In Fig. 2, we show that the bounded motion conditions are met: the oscillatory behavior of the nodal period $T_{\mathrm{d}}$ and the ascending node drift $\Delta \Omega$ of the two orbits $\mathcal{O}_{1}$ and $\mathcal{O}_{2}$ averages out to the same value, respectively, which corresponds to the constant nodal period $T_{\mathrm{d}}^{\star}$ and constant ascending node drift $\Delta \Omega^{\star}$ of the fixed-point orbit $\mathcal{O}_{0}$.

The bounded motion is further confirmed by Fig. 3, which shows the total distance between the three LEOs, respectively, for 14 years. Furthermore, Fig. 3 illustrates the relative radial and along-track distance between the orbit pairs from the perspective of one of the orbits in the pair.

Apart from yielding long-term bounded motion, the normal form methods also provide the average angle advancement $\Lambda$ in the $\left(r, v_{r}\right)$ phase space. This angle advancement is directly linked to the rotation frequency $\omega_{p}$ of the orbit (and its apsides) within its orbital plane, which causes the oscillation of $T_{\mathrm{d}}$ and $\Delta \Omega$ shown in Fig. 2 with $\omega_{p}$. One $\left(r, v_{r}\right)$ phase space rotation corresponds to one revolution of the orbit (and its apsides) within its orbital plane. Accordingly, the frequency $\omega_{p}=\Lambda / 2 \pi$ is equivalent to the definition of the tune and the tune shifts $v+\delta v$, which are just the normalized angle advancement separated into its constant 

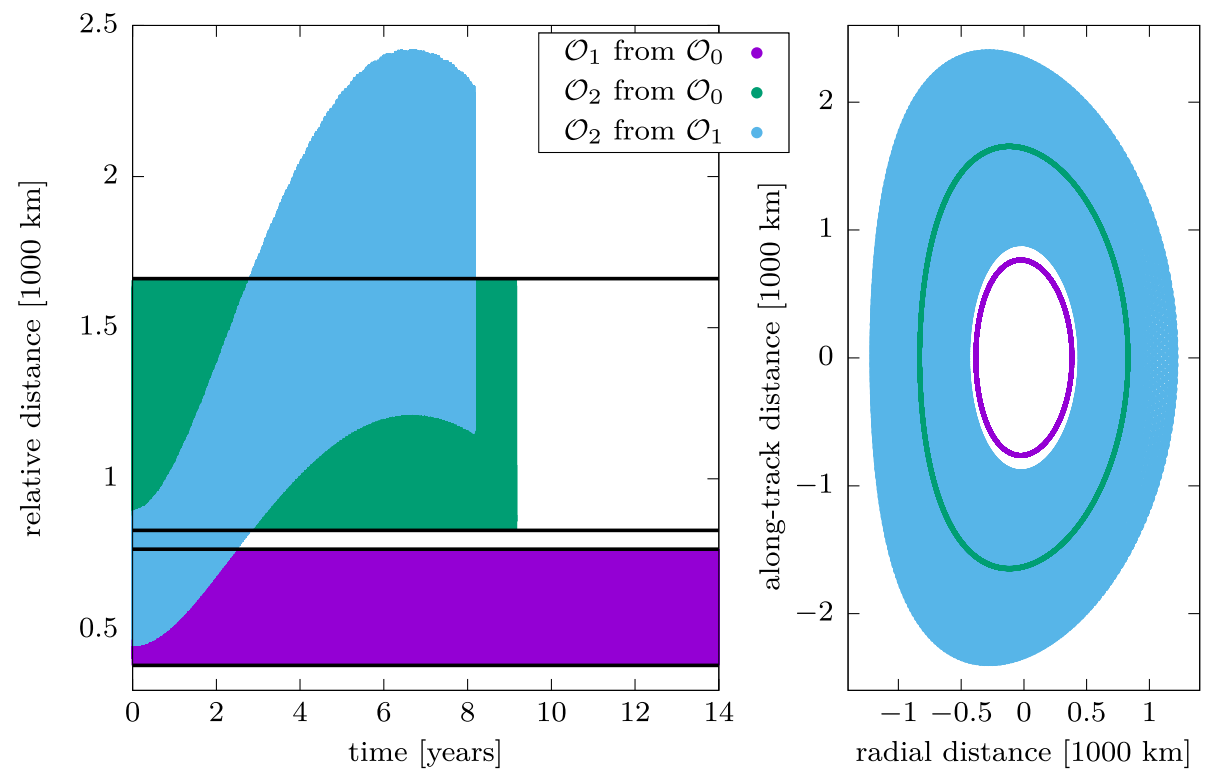

Fig. 3 Relative bounded motion of LEOs with an average nodal period of $\overline{T_{d}}=7.64916169(\approx 103 \mathrm{~min})$ and an average node drift of $\overline{\Delta \Omega}=1.22871195 \mathrm{E}-3 \mathrm{rad}$ for 14 years. The total relative distance between the orbits is shown in the left plot and the right plot shows the relative radial and along-track distance between orbit pairs from the perspective of one of the orbits in the pair. The oscillation in the relative distance between $\mathcal{O}_{2}$ and $\mathcal{O}_{1}$ is caused by the rotating orbital orientation of the orbits at different frequencies as explained in the text

Table 3 Expansion of $\omega_{p}\left(\delta r, \delta v_{r}=0\right)$ of relative bounded motion LEOs with an average nodal period $\overline{T_{d}}=7.64916169(\approx 103 \mathrm{~min})$ and an average node drift of $\overline{\Delta \Omega}=1.22871195 \mathrm{E}-3 \mathrm{rad}$. The expansion is relative to the pseudo-circular LEO from $\mathrm{He}$ et al. (2018)

\begin{tabular}{lc}
\hline$\omega_{p}\left(\delta r, \delta v_{r}=0\right)=$ & \\
\hline$+0.54868728 \mathrm{E}-3$ & \\
$+0.10803872 \mathrm{E}-2$ & $\delta r^{2}$ \\
$+0.86800515 \mathrm{E}-6$ & $\delta r^{3}$ \\
$+0.10552068 \mathrm{E}-2$ & $\delta r^{4}$ \\
$+0.29106874 \mathrm{E}-5$ & $\delta r^{5}$ \\
$-0.76284414 \mathrm{E}-3$ & $\delta r^{6}$ \\
$+0.39324207 \mathrm{E}-5$ & $\delta r^{7}$ \\
$-0.35077526 \mathrm{E}-1$ & $\delta r^{8}$ \\
\hline
\end{tabular}

part (the tune $v$ ) and its amplitude-dependent part (the tune shifts $\delta v$ ). The normal form yields the average angle advancement $\Lambda$ dependent on $\left(r_{\mathrm{NF}}, \delta \mathcal{H}_{z}, \delta E\right)$. After normalizing $\Lambda$, by division by $2 \pi$, replacing $r_{\mathrm{NF}}$ by an expression of $\left(\delta r, \delta v_{r}\right)$ and $\left(\delta \mathcal{H}_{z}, \delta E\right)$ according to Eq. 40 and using the expressions from Table 9 for $\left(\delta \mathcal{H}_{z}, \delta E\right)$, the frequency $\omega_{p}\left(\delta r, \delta v_{r}\right)$ is obtained for the bounded motion orbits around the fixed-point LEO (see Table 9). The coefficients of $\omega_{p}$ for $\delta v_{r}=0$ are given in Table 3 .

Accordingly, the periods of the oscillations of the nodal periods $T_{\mathrm{d}}$ and the ascending node drifts $\Delta \Omega$ in Fig. 2 (in units of orbital revolutions) are just the inverse of the frequencies $\omega_{p}(\delta r=0.06)=5.52590498 \mathrm{E}-4$ and $\omega_{p}(\delta r=0.13)=5.67242676 \mathrm{E}-4$. These frequencies 
also help explain the oscillation of the total relative distance range between $\mathcal{O}_{1} \rightleftarrows \mathcal{O}_{2}$ over 13.3 years in Fig. 3.

While $\mathcal{O}_{1}$ shows repetitive behavior after 1809.7 orbital revolutions (129.3 days), the behavior of $\mathcal{O}_{2}$ is repetitive after 1762.9 orbital revolutions (125.9 days). Accordingly, the two orbits will be in and out of sync regarding their orbital orientation, while maintaining bounded due to the matching average nodal period and ascending node drift. Specifically, the two orbits will be back in sync after about 68170 orbital revolutions (4869 days/13.3 years) as illustrated in Fig. 3, since $\mathcal{O}_{1}$ will have turned 37.7 times, while $\mathcal{O}_{2}$ will have turn exactly once less, namely 36.7 times, bringing them both back into the same orbital orientation to one another before moving apart again.

In conclusion, our first comparison showed the superiority of the normal form methods, particularly compared to the iterative map evaluation method in He et al. (2018), where numerical adjustments to the method were required to provide long-term relative bounded motion for $\delta r=0.11$.

In Sect. 5.3, we will show that the DANF method even provides hypothetical long-term bounded motion up to $\delta r=0.3$, which covers all realistic cases until $\delta r=0.14$ and further hypothetical (non-practical) cases with altitudes below the Earth's surface.

In the next comparison, we are going to investigate bounded motion much farther from the Earth's surface. Accordingly, we expect a larger theoretical and practical bounded motion range from the DANF method, due to a weaker influence of the zonal perturbations.

\subsection{Bounded motion in medium Earth orbit}

In this comparison, we are considering a medium Earth orbit (MEO) from Baresi and Scheeres (2017b, p.11) with $r=26562.58 \mathrm{~km}, v_{r}=-9.05 \mathrm{E}-4 \mathrm{~km} / \mathrm{s}$ and $v_{z}=3.18 \mathrm{~km} / \mathrm{s}$. In the units of $R_{0}=6378.137 \mathrm{~km}$ and $T_{0}=806.811 \mathrm{~s}$, the zonal problem with $J_{2}$ to $J_{15}$ yields a fixed-point orbit at $\left(r^{\star}, v_{r}^{\star}\right)=(4.17198963,-1.14150072 \mathrm{E}-4)$ and $v_{z}^{\star}=0.40154964$ for the parameters $\left(\mathcal{H}_{z}, E\right)=(1.16863390,-0.11984818)$. The fixed-point orbit has a fixed nodal period $T_{d}^{\star}=53.5395648(\approx 12$ hours $)$ and constant drift in the ascending node of $\Delta \Omega^{\star}=-3.35410945 \mathrm{E}-4 \mathrm{rad}\left(-0.0192^{\circ}\right)$.

The computation of the map took $131 \mathrm{~s}$ on the same computer system as mentioned above (Sect. 5.1). The offset of the integration with $\left(\Delta r, \Delta v_{r}, \Delta z, \Delta v_{z}\right)=$ $(-4 \mathrm{E}-15,-2 \mathrm{E}-13,-4 \mathrm{E}-15,2 \mathrm{E}-16)$ is well within the range of the numerical error of the integration. After the normal form transformation (in $100 \mathrm{~ms}$ ) and the averaging (in $62 \mathrm{~ms}$ ) following the same procedure as in Sect. 5.1, the dependencies of the constants of motion $\left(\mathcal{H}_{z}, E\right)$ on $\left(\delta r, \delta v_{r}\right)$ were calculated (see Table 10 in "Appendix"). Table 4 yields $\mathcal{H}_{z}\left(\delta r, \delta v_{r}=0\right)$ and $E\left(\delta r, \delta v_{r}=0\right)$.

To illustrate that the DANF methods also provide bounded motion for this set of parameters, we consider the long-term behavior of three MEOs relative to one another. The first orbit is the fixed-point/pseudo-circular orbit and is denoted with $\mathcal{O}_{0}$. Since $r^{\star}$ of the fixed-point MEO is about four times the $r^{\star}$ of the low Earth fixed-point orbit from the previous section, the bounded orbits are initiated at four times the distance compared to the LEO investigation in Sect. 5.1. $\mathcal{O}_{1}$ is initiated at $\delta r=0.24(1531 \mathrm{~km})$ with $\delta v_{r}=0$ and $\mathcal{O}_{2}$ is initiated at $\delta r=0.52(3317 \mathrm{~km})$ with $\delta v_{r}=0$. These relative distances are already at the border or larger than distances that are used in practice. Again, both orbits have an initial longitudinal offset of $\phi=0.5^{\circ}$ relative to $\mathcal{O}_{0}$. The specific values of the orbits are given in Table 5 .

Equivalent to Fig. 2, we show that the bounded motion conditions are met for the chosen MEOs in Fig 4. The oscillatory behavior of the nodal period $T_{\mathrm{d}}$ and the ascending node 
Table 4 Expansion of

$\mathcal{H}_{z}\left(\delta r, \delta v_{r}=0\right)$ and

$E\left(\delta r, \delta v_{r}=0\right)$ for relative

bounded motion MEOs with an

average nodal period of

$\overline{T_{d}}=53.5395648(\approx 12 \mathrm{~h})$ and

an average node drift of

$\overline{\Delta \Omega}=-3.35410945 \mathrm{E}-4 \mathrm{rad}$. The

expansion is relative to the

pseudo-circular MEO from

Baresi and Scheeres (2017b)

\begin{tabular}{llll}
\hline $\mathcal{H}_{z}\left(\delta r, \delta v_{r}=0\right)=$ & & $E\left(\delta r, \delta v_{r}=0\right)=$ \\
\hline+1.16863390 & & -0.11984818 & \\
-0.16787983 & $\delta r^{2}$ & $-0.11295792 \mathrm{E}-05$ & $\delta r^{2}$ \\
$-0.57819536 \mathrm{E}-5$ & $\delta r^{3}$ & $-0.38903865 \mathrm{E}-10$ & $\delta r^{3}$ \\
$+0.72342680 \mathrm{E}-2$ & $\delta r^{4}$ & $-0.16786161 \mathrm{E}-07$ & $\delta r^{4}$ \\
$+0.16208617 \mathrm{E}-6$ & $\delta r^{5}$ & $-0.34176382 \mathrm{E}-11$ & $\delta r^{5}$ \\
$-0.69493130 \mathrm{E}-4$ & $\delta r^{6}$ & $-0.28279909 \mathrm{E}-08$ & $\delta r^{6}$ \\
$+0.11561378 \mathrm{E}-6$ & $\delta r^{7}$ & $+0.27190622 \mathrm{E}-12$ & $\delta r^{7}$ \\
$+0.54888817 \mathrm{E}-4$ & $\delta r^{8}$ & $-0.51224108 \mathrm{E}-10$ & $\delta r^{8}$ \\
\hline
\end{tabular}

Table 5 All MEOs are initiated with $v_{r, 0}=-1.14150072 \mathrm{E}-4$ and $r_{0}=4.17198963+\delta r$ and have an average nodal period of $\bar{T}_{d}=53.5395648(\approx 12 \mathrm{~h})$ and an average ascending node drift of $\overline{\Delta \Omega}=-3.35410945 \mathrm{E}-4$ $\mathrm{rad} . \mathcal{O}_{0}$ is the pseudo-circular MEO from Baresi and Scheeres (2017b)

\begin{tabular}{llllll}
\hline & $\delta r$ & $\delta v_{r}$ & $\phi$ & $\mathcal{H}_{z}$ & \multicolumn{1}{c}{$E$} \\
\hline $\mathcal{O}_{0}$ & 0.0 & 0 & $0.0^{\circ}$ & 1.16863390 & -0.119848175 \\
$\mathcal{O}_{1}$ & $0.24(1531 \mathrm{~km})$ & 0 & $0.5^{\circ}$ & 1.15898794 & -0.119848240 \\
$\mathcal{O}_{2}$ & $0.52(3317 \mathrm{~km})$ & 0 & $0.5^{\circ}$ & 1.123766254 & -0.119848482 \\
\hline
\end{tabular}

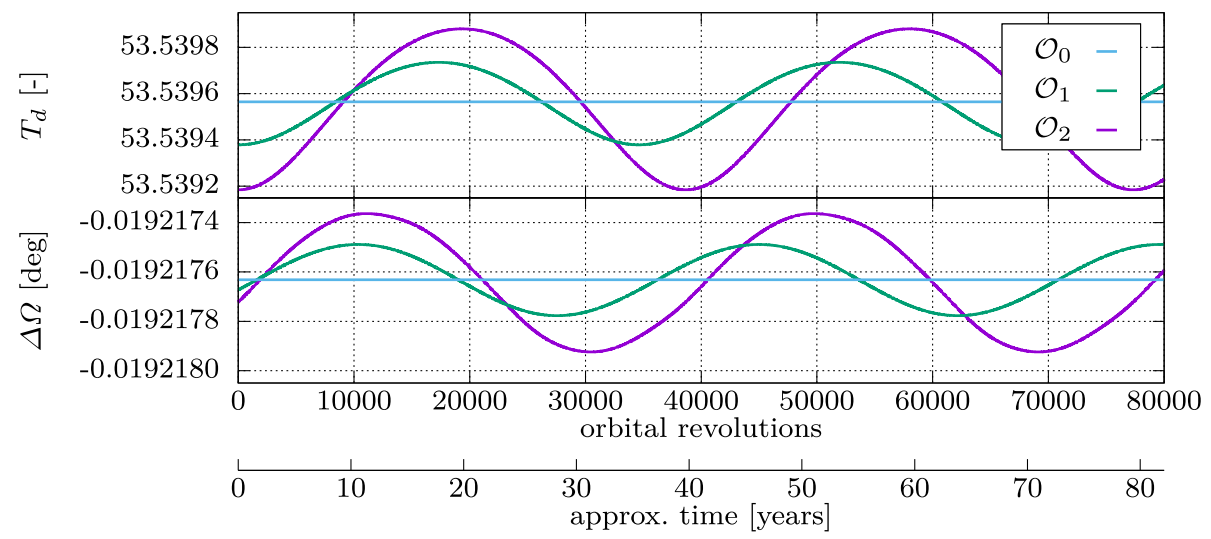

Fig. 4 Oscillatory behavior of the bounded motion quantities $T_{\mathrm{d}}$ and $\Delta \Omega$ of the bounded MEOs $\mathcal{O}_{1}$ and $\mathcal{O}_{2}$ initiated at $\delta r=0.24$ and $\delta r=0.52$, respectively. Additionally, the constant nodal period $T_{d}^{\star}=53.5395648$ and constant ascending node drift of $\Delta \Omega^{\star}=-0.0192176316 \mathrm{deg}$ of the fixed-point orbit $\mathcal{O}_{0}$ are shown. The periods of oscillation are 38682 orbital revolutions (52.9 years) for $\mathcal{O}_{2}, 34621$ orbital revolutions (47.4 years) for $\mathcal{O}_{1}$ and 33671 orbital revolutions (46.1 years) for $\delta r \rightarrow 0$ of $\mathcal{O}_{0}$. The shown results are generated by numerical integration. The time domain was added, assuming that on average one orbital revolution $\hat{\approx} T_{\mathrm{d}}^{\star}$

drift $\Delta \Omega$ of the two orbits $\mathcal{O}_{1}$ and $\mathcal{O}_{2}$ averages out to the same values, respectively, which correspond to the constant nodal period $T_{\mathrm{d}}^{\star}$ and constant ascending node drift $\Delta \Omega^{\star}$ of the fixed-point orbit $\mathcal{O}_{0}$. In contrast to the investigated LEOs, the oscillation period of the bounded motion quantities of the MEOs increases with increasing $\delta r$. The period of oscillation in the MEO cases is also about two orders of magnitude longer with periods of 47 and 53 years for $\mathcal{O}_{1}$ and $\mathcal{O}_{2}$, respectively, compared to the LEOs. 
Table 6 Expansion of

$\omega_{p}\left(\delta r, \delta v_{r}=0\right)$ of relative bounded motion orbits with an average nodal period of

$\bar{T}_{d}=53.5395648(\approx 12 \mathrm{~h})$ and an average ascending node drift of $\overline{\Delta \Omega}=-3.35410945 \mathrm{E}-4 \mathrm{rad}$. The expansion is relative to the pseudo-circular MEO from Baresi and Scheeres (2017b)

\begin{tabular}{ll}
\hline$\omega_{p}\left(\delta r, \delta v_{r}=0\right)=$ & \\
\hline$+0.29699500 \mathrm{E}-04$ & \\
$-0.14137545 \mathrm{E}-04$ & $\delta r^{2}$ \\
$-0.48691156 \mathrm{E}-09$ & $\delta r^{3}$ \\
$-0.22644327 \mathrm{E}-06$ & $\delta r^{4}$ \\
$-0.43912160 \mathrm{E}-10$ & $\delta r^{5}$ \\
$-0.10717280 \mathrm{E}-05$ & $\delta r^{6}$ \\
$-0.10374073 \mathrm{E}-09$ & $\delta r^{7}$ \\
$+0.23789772 \mathrm{E}-05$ & $\delta r^{8}$
\end{tabular}
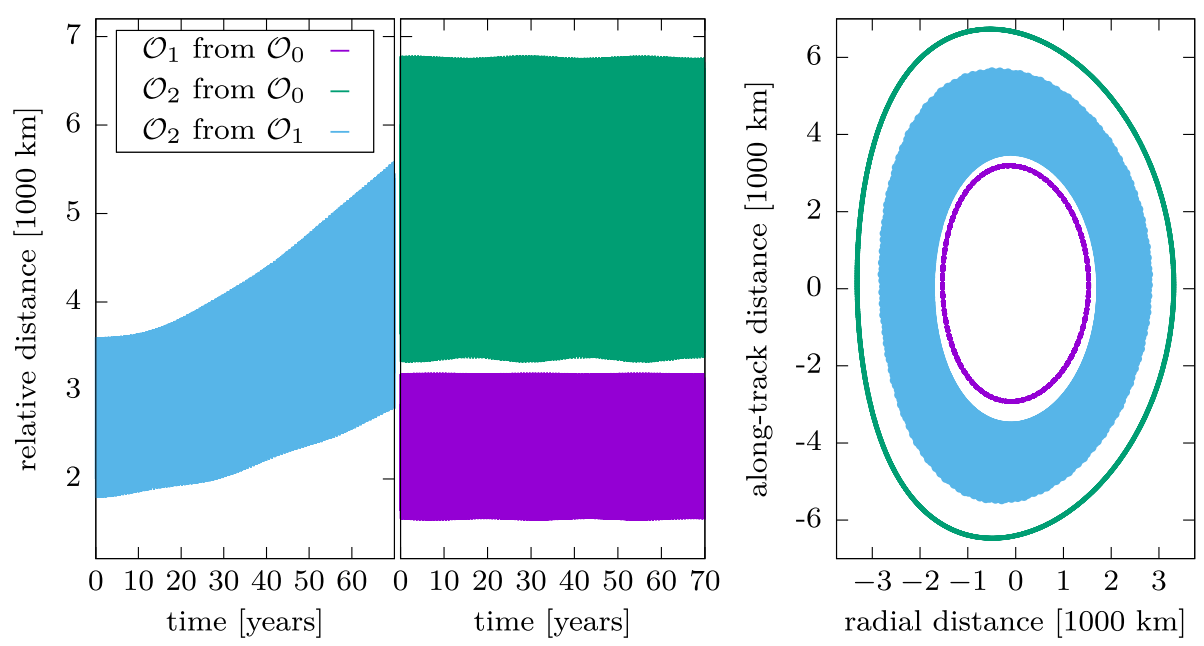

Fig. 5 Relative bounded motion of MEOs with an average nodal period of $\overline{T_{d}}=53.5395648(\approx 12 \mathrm{~h})$ and an average ascending node drift of $\overline{\Delta \Omega}=-3.35410945 \mathrm{E}-4$ rad over 70 years. The total relative distance between the orbits is shown in the left plots, and the right plot shows the relative radial and along-track distance between orbit pairs from the perspective of one of the orbits in the pair. The 'breathing' of the relative total distance between $\mathcal{O}_{2}$ and $\mathcal{O}_{0}$ originates from the rotating orbital orientation of pseudo-elliptical $\mathcal{O}_{2}$ relative to the pseudo-circular $\mathcal{O}_{0}$. Due to the very long rotation periods, only the first 70 years of the relative distance oscillation and radial/along-track behavior between $\mathcal{O}_{2}$ and $\mathcal{O}_{1}$ could be shown

Using the normal form methods, the rotation frequency $\omega_{p}$ of the orbital orientation within its orbital plane is calculated as described in Sect. 5.1. The results from the expansion of $\omega_{p}$ confirm these periods of oscillation with $\omega_{p}(0.24)=2.88842404 \mathrm{E}-5$ and $\omega_{p}(0.52)=$ $2.58516089 \mathrm{E}-5$. The expansion of $\omega_{p}$ dependent on $\delta r$ is given in Table 6 . The full expansion $\omega_{p}\left(\delta r, \delta v_{r}\right)$ is provided in Table 10 in "Appendix."

Figure 5 shows the long-term bounded motion behavior by illustrating the relative total distance between the orbits and their relative radial and along-track distances. Due to the long oscillation periods in the bounded motion quantities of 47 and 53 years for $\mathcal{O}_{1}$ and $\mathcal{O}_{2}$, respectively, the oscillation in the total distance between $\mathcal{O}_{1}$ and $\mathcal{O}_{2}$ is about 456 years and can therefore only be partially shown. After 456 years, the orbital orientation of $\mathcal{O}_{1}$ will have turned 9.6 times and align again with the orbital orientation of $\mathcal{O}_{2}$, which will have turned 8.6 times. 
Table 7 The following orbit parameters are obtained by evaluating $\mathcal{H}_{z}\left(\delta r, \delta v_{r}=0\right)$ and $E\left(\delta r, \delta v_{r}=0\right)$ from Tables 1 and 4 for various $\delta r$ keeping $\delta v_{r}=0$

\begin{tabular}{|c|c|c|c|c|c|c|c|}
\hline \multicolumn{4}{|c|}{ Test LEOs } & \multicolumn{4}{|c|}{ Test MEOs } \\
\hline & $\delta r$ & $\mathcal{H}_{z}$ & $E$ & & $\delta r$ & $\mathcal{H}_{z}$ & $E$ \\
\hline $\mathcal{O}_{0}$ & 0.00 & -0.167072950 & -0.438705274 & $\mathcal{O}_{0}$ & 0.0 & 1.16863390 & -0.119848175 \\
\hline $\mathcal{O}_{0.15}$ & 0.15 & -0.159952468 & -0.438712546 & $\mathcal{O}_{0.6}$ & 0.6 & 1.10913117 & -0.119848584 \\
\hline $\mathcal{O}_{0.20}$ & 0.20 & -0.154547603 & -0.438718435 & $\mathcal{O}_{0.7}$ & 0.7 & 1.08810278 & -0.119848733 \\
\hline $\mathcal{O}_{0.25}$ & 0.25 & -0.147770789 & -0.438726324 & $\mathcal{O}_{0.8}$ & 0.8 & 1.06414208 & -0.119848906 \\
\hline $\mathcal{O}_{0.30}$ & 0.30 & -0.139754169 & -0.438736486 & $\mathcal{O}_{0.9}$ & 0.9 & 1.03738028 & -0.119849103 \\
\hline $\mathcal{O}_{0.35}$ & 0.35 & -0.130665569 & -0.438749297 & $\mathcal{O}_{1.0}$ & 1.0 & 1.00796823 & -0.119849324 \\
\hline \multirow[t]{4}{*}{$\mathcal{O}_{0.40}$} & 0.40 & -0.120716695 & -0.438765264 & $\mathcal{O}_{1.1}$ & 1.1 & 0.976078338 & -0.119849572 \\
\hline & & & & $\mathcal{O}_{1.2}$ & 1.2 & 0.941907258 & -0.119849845 \\
\hline & & & & $\mathcal{O}_{1.3}$ & 1.3 & 0.905679721 & -0.119850146 \\
\hline & & & & $\mathcal{O}_{1.4}$ & 1.4 & 0.867653615 & -0.119850476 \\
\hline
\end{tabular}

The 'breathing' of the relative distance between the orbits is particularly noticeable for the orbit pair of $\mathcal{O}_{2}$ and $\mathcal{O}_{0}$. The frequency of the 'breathing' is $2 \omega_{p}$ which is a result of the rotation of the orbital orientation of the pseudo-elliptical $\mathcal{O}_{2}$ relative to the pseudo-circular $\mathcal{O}_{0}$. Since the orbital shape of the pseudo-elliptical $\mathcal{O}_{2}$ is approximately symmetric along its semi-major axis, one full rotation of the orbital orientation corresponds to two breathing cycles.

In conclusion, our methods also provided an entire set of long-term relative bounded motion around the considered fixed-point MEO from Baresi and Scheeres (2017b), which was validated far beyond practical relative distances. In the following subsection, the limitations of our method are investigated. The investigations will show that the validity of the sets presented in Sects. 5.1 and 5.2 extends over about twice the already-presented distance from their respective fixed-point orbits.

\subsection{Testing the limitations of the DANF method}

The previous two sections illustrate the validity of the DANF method for all practical relative distances for bounded motion and beyond. In this section, we move even further away from any practical relevance of the calculated sets of bounded motion to the limitations of our method. Since it is based on polynomial expansions, it is obvious it will fail at some point and we want to show when and how this failing process takes place.

First, we pick a number of test orbits from the calculated bounded motion sets (see Table 7). In contrast to previous examples, no initial longitudinal offset relative to the respective fixedpoint orbits is set.

Figure 6 illustrates the behavior of the bounded motion quantities $T_{\mathrm{d}}$ and $\Delta \Omega$ for the chosen orbits of the LEO-bounded motion set. Both quantities show oscillatory behavior centered at or close to $T_{\mathrm{d}}^{\star}$ and $\Delta \Omega^{\star}$, respectively. With increasing distance $\delta r$, the influence of higher-order oscillations becomes apparent. The frequency and amplitude of oscillation of the bounded motion quantities also increase with increasing distance $\delta r$.

If the bounded motion conditions are not met or only met approximately, the orbits will start drifting apart. This effect is illustrated in Fig. 7, which shows very slowly diverging 


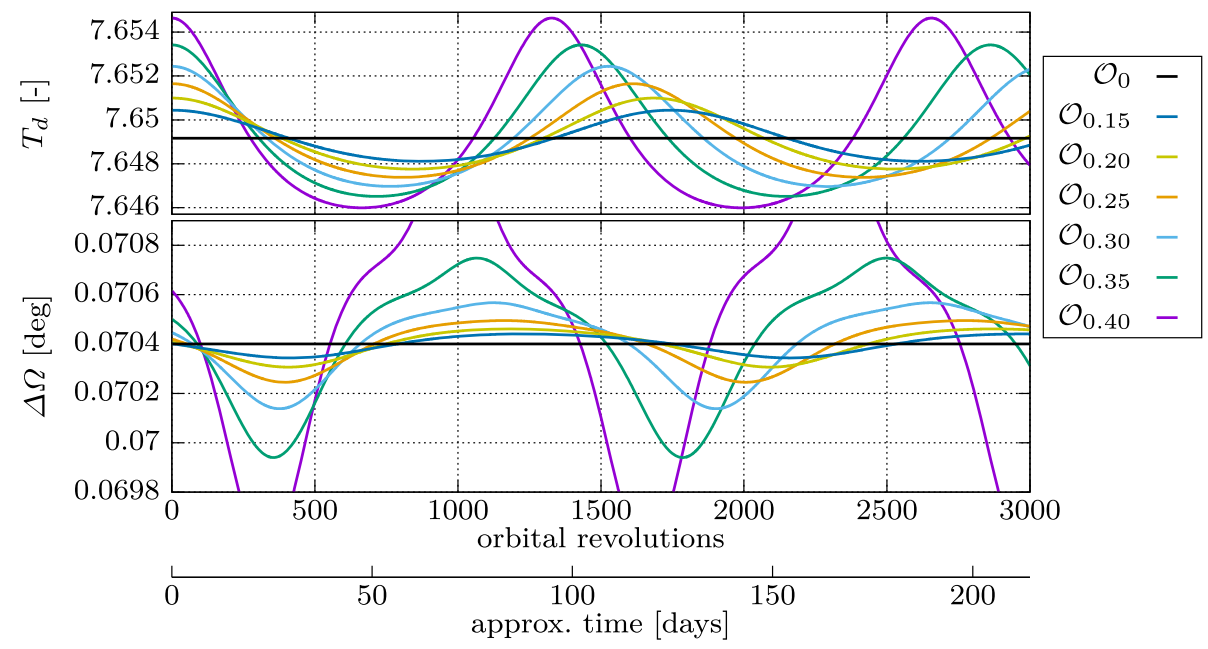

Fig. 6 Behavior of the bounded motion quantities $T_{\mathrm{d}}$ and $\Delta \Omega$ for the test orbits from Table 7 of the calculated LEO-bounded motion set (see Table 9) generated by numerical integration. For large $\delta r$, the influences of higher-order oscillations are apparent. The frequency and amplitude of oscillation increase with increasing $\delta r$. The amplitude of $\Delta \Omega$ is particularly sensitive to $\delta r$

behavior of approximately $2.6 \mathrm{~km} /$ year for $\delta r=0.3(1913 \mathrm{~km})$ and a stronger divergence of approximately $10.6 \mathrm{~km} /$ year for $\delta r=0.4(2551 \mathrm{~km})$ in the left plot. The thickening curves in the radial/along-track representation of the relative orbit motion are further indication of divergence. The strength of divergence in Fig. 7 can be directly linked to the size of the offsets in the bounded motion quantities from $T_{\mathrm{d}}^{\star}$ and $\Delta \Omega^{\star}$, as shown in Fig. 6.

From Figs. 7 and 6, we conclude that our method and the resulting expansions in $\mathcal{H}_{z}$ and $E$ (see Table 9) for long-term bounded motion of at least 10 years around the fixed-point LEO from He et al. (2018) start to lose their significant accuracy for $\delta r \geq 0.3$ to satisfy the bounded motion conditions with the required precision. Note that $\delta r=0.3(1913 \mathrm{~km})$ is already a purely theoretical orbit with altitudes of more than $1000 \mathrm{~km}$ below the Earth's surface, which means that our expansions in $\mathcal{H}_{z}$ and $E$ provided reliable bounded motion beyond realistic $(\delta r \leq 0.14)$ inter-orbit distances.

The behavior of the bounded motion quantities $T_{\mathrm{d}}$ and $\Delta \Omega$ for the chosen orbits of the MEO-bounded motion set (Table 7) is shown in Fig. 8. In contrast to the test LEOs, the amplitude and period of oscillation of the bounded motion quantities are decreasing with increasing distance $\delta r$, which causes the almost steady behavior of $\delta r=1.4$ over the shown time span and generally suppresses higher-order oscillations that were seen for the LEOs. While the oscillations of $T_{\mathrm{d}}$ are approximately centered around $T_{\mathrm{d}}^{\star}$ (with the exception of $\mathcal{O}_{1.4}$ ), the center of oscillation is increasingly diverging from $\Delta \Omega^{\star}$ to lower $\Delta \Omega$ for $\delta r \geq 0.8$. In other words, the expansions in $\delta \mathcal{H}_{z}$ and $\delta E$ start failing in producing related orbits that satisfy the bounded motion condition.

The consequence of this offset in the bounded motion condition is diverging behavior between the orbits, which is shown in Fig. 9. The upper bound of the total distance between the orbits starts diverging for those very large distances, and the thickening curves in the radial/along-track representation of the orbits distance from the perspective of $\mathcal{O}_{0}$ further indicate this divergence. Additionally, Fig. 9 shows the 'breathing' in the total relative dis- 

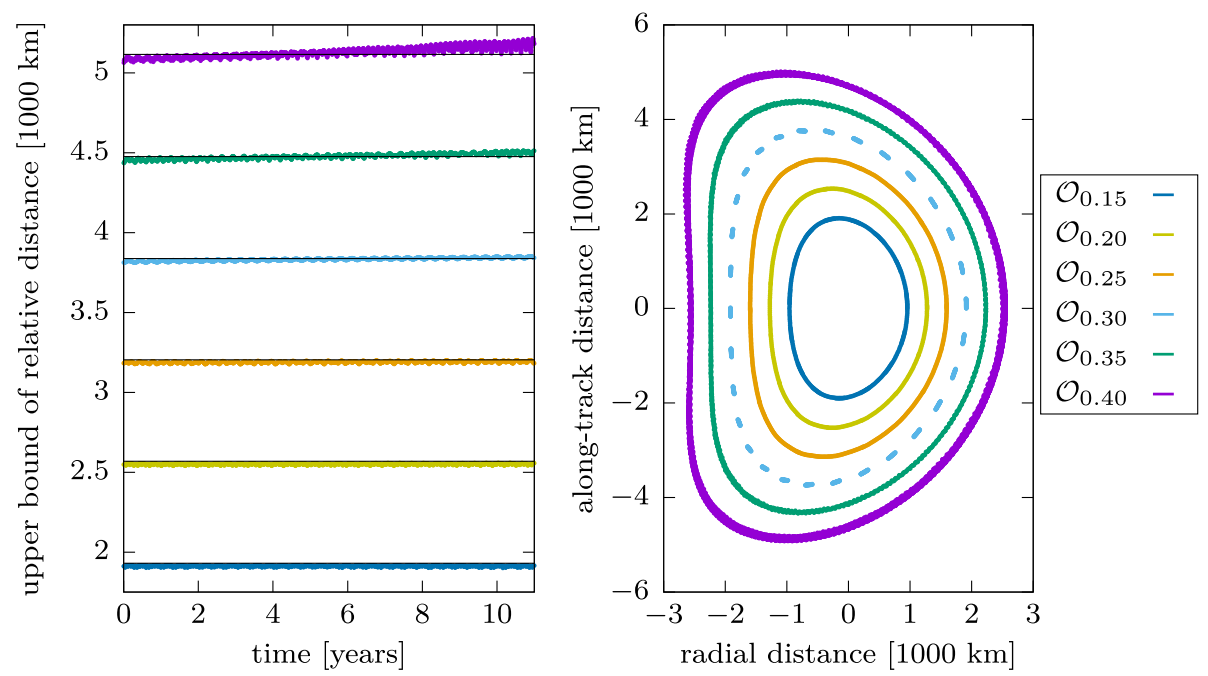

Fig. 7 Distance between the orbits in the calculated bounded motion set and $\mathcal{O}_{0}$ is determined in regular time intervals with numerical integration over more than 10 years. Left plot only shows the upper bound to avoid overlaps. Thin horizontal lines at initial upper bound emphasize small changes. The dotted light blue curve (right) originates from an unintended near resonance between the chosen time interval for distance evaluations and the orbital behavior. A measurable increase in relative distances (left) over 10 years for $\delta r \geq 0.3$ is supported by thickening curves in the radial/along-track behavior (right)

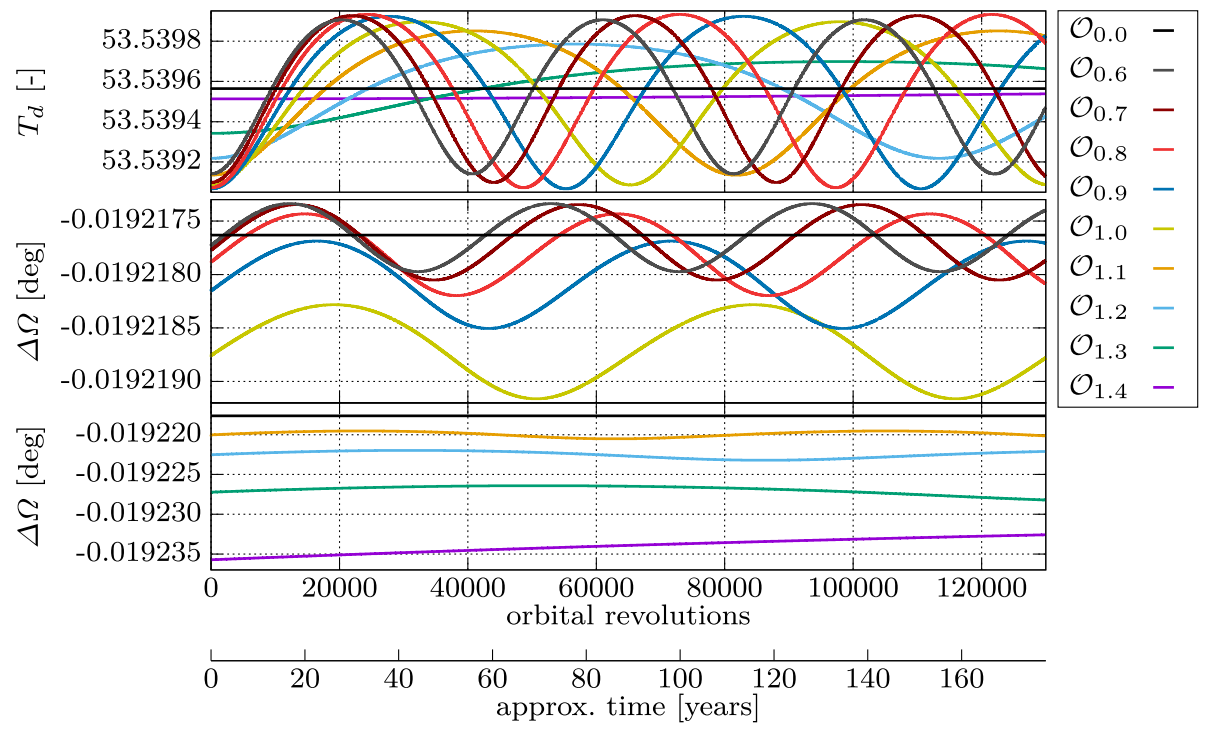

Fig. 8 Behavior of the bounded motion quantities $T_{\mathrm{d}}$ and $\Delta \Omega$ for the test orbits from Table 7 of the calculated MEO-bounded motion set (see Table 10) generated by numerical integration. In contrast to the investigated LEOs, the frequency and amplitude of oscillation decrease with increasing $\delta r$ such that $\mathcal{O}_{1.4}$ appears almost steady. For $\delta r \geq 0.8$, the center of oscillation of $\Delta \Omega$ starts to drift to more negative values and away from $\Delta \Omega^{\star}$ 

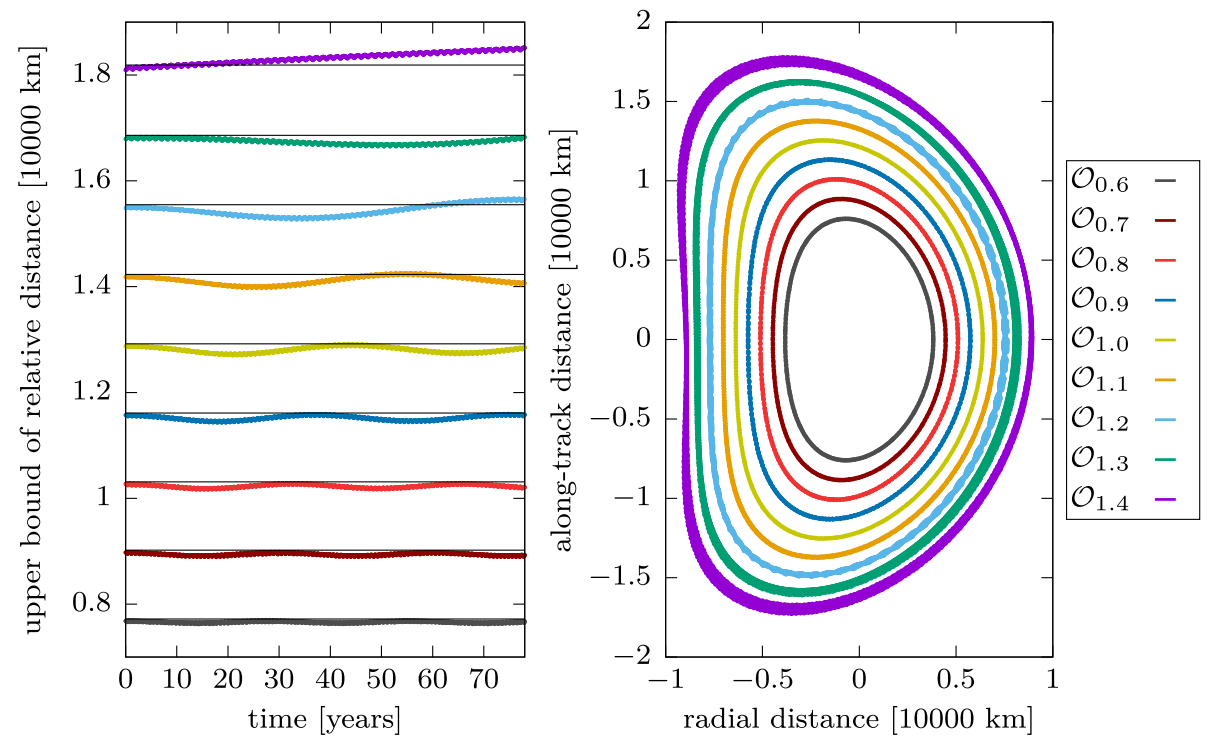

Fig. 9 Distance between the orbits in the calculated bounded motion set and $\mathcal{O}_{0}$ is determined in regular time intervals by numerical integration over more than 70 years. Left plot only shows the upper bound to avoid overlaps. Thin horizontal lines at initial upper bound emphasize small changes. The 'breathing' of the total relative distance from the orbital rotation is clearly visible. Its period increases with increasing $\delta r$ until being unrecognizable due to the strong divergence for $\delta r \geq 1.4$, which is supported by thinker curves in the right plot. Weaker divergence over the 70-year time span is already noticeable for $\delta r \geq 0.9$. The divergence is caused by the offset in respective bounded motion quantities (see Fig. 8)

tance between the orbits with $2 \omega_{p}$, which is due to the rotating orbital orientation of the orbits relative to the pseudo-circular fixed-point orbit as already mentioned in the section above.

From Figs. 9 and 8, we conclude that our method and the resulting expansions in $\mathcal{H}_{z}$ and $E$ (see Table 9) for long-term bounded motion of at least 70 years around the fixed-point MEO from Baresi and Scheeres (2017b) start to lose their significant accuracy for $\delta r \geq 0.9$ to satisfy the bounded motion conditions with the required precision. Interestingly, the very long 'breathing' periods for very large distances like $\delta r=1.3$ suggested (temporary) bounded motion for the first 70 years when looking at Fig. 9, while Fig. 8 reveals the underlying diverging behavior due to the mismatched bounded motion conditions.

\section{Conclusion}

The normal form methods presented in this work yield parameterized sets of the constants of motion $\left(\mathcal{H}_{z}(\delta r), E(\delta r)\right)$ for bounded orbits with an average nodal period and average ascending node drift corresponding to the fixed nodal period and ascending node drift of the reference (fixed-point) orbit. The range of $\delta r$ for which bounded motion orbits can be obtained is dependent on the closeness to the Earth. The closer to the Earth, the stronger the influence of the zonal perturbation and the stronger the dynamics of the orbit relatively depend on $\delta r$.

In comparison with the approach in He et al. (2018), our method avoided the timeconsuming and inaccurate numerical averaging, by using a normal form based parameteriza- 
tion for the averaging. As a result, the range of the bounded motion provided by our methods is more than twice as large as the range of the results in He et al. (2018). Additionally, our method does not require a separate calculation for each $\delta r$, but rather provides an expansion in $\left(\delta r, \delta v_{r}\right)$, which covers all orbits up to a certain maximum range that varies with the altitude of the reference trajectory (see Sect. 5).

While the method in Baresi and Scheeres (2017b) has the advantage of allowing for the calculation of bounded orbits up to arbitrary distances $\delta r$, it lacks the ability to provide parameterized sets of bounded motion just like He et al. (2018).

The normal form methods are also able to provide parameterized sets of the rotation frequency of the orbits within their orbital plane. This rotation is due to the zonal perturbations in the gravitational field of the Earth, since there is no rotation of the orbit for the spherically symmetric case. With increasing distance from the Earth's center $\rho$, the zonal perturbations $J_{l}$ fall off with $\rho^{-l-1}$. Accordingly, it is not surprising that the rotation frequency of the MEOs is so much lower than the rotation frequency of the LEOs. Similarly, the $\delta r$ dependence of the bounded motion is a lot less sensitive for the MEOs compared to the LEOs.

Future efforts can be dedicated to use our methods for more practical mission design problem, thus including additional perturbations, control and verified results.

Acknowledgements This work was supported by the Studienstiftung des deutschen Volkes with a scholarship to one of the authors (A. Weisskopf). The foundation of the normal form methods has been developed for beam physics application under the contract no. DE-FG02-08ER41546/DE-SC0018636 of the U.S. Department of Energy. Additionally, we greatly appreciate the insightful comments from the two reviewers of this paper.

\section{Compliance with ethical standards}

Conflict of interest The authors declare that there is no conflict of interest.

\section{Appendix}

See Tables 8, 9 and 10 .

Table 8 Used values for coefficients $J_{2}$ to $J_{15}$

\begin{tabular}{ll}
\hline$J_{2}$ & $0.108265 \mathrm{E}-2$ \\
$J_{3}$ & $-0.253198197166358 \mathrm{E}-5$ \\
$J_{4}$ & $-0.162044603134295 \mathrm{E}-5$ \\
$J_{5}$ & $-0.227974752444200 \mathrm{E}-6$ \\
$J_{6}$ & $0.541020690186084 \mathrm{E}-6$ \\
$J_{7}$ & $-0.350290712062631 \mathrm{E}-6$ \\
$J_{8}$ & $-0.203807006663452 \mathrm{E}-6$ \\
$J_{9}$ & $-0.121996071986768 \mathrm{E}-6$ \\
$J_{10}$ & $-0.244590838844460 \mathrm{E}-6$ \\
$J_{11}$ & $0.243134750456128 \mathrm{E}-6$ \\
$J_{12}$ & $-0.182033357729771 \mathrm{E}-6$ \\
$J_{13}$ & $-0.216491043170781 \mathrm{E}-6$ \\
$J_{14}$ & $0.122216071480691 \mathrm{E}-6$ \\
$J_{15}$ & $-0.125428247724183 \mathrm{E}-7$ \\
\hline
\end{tabular}




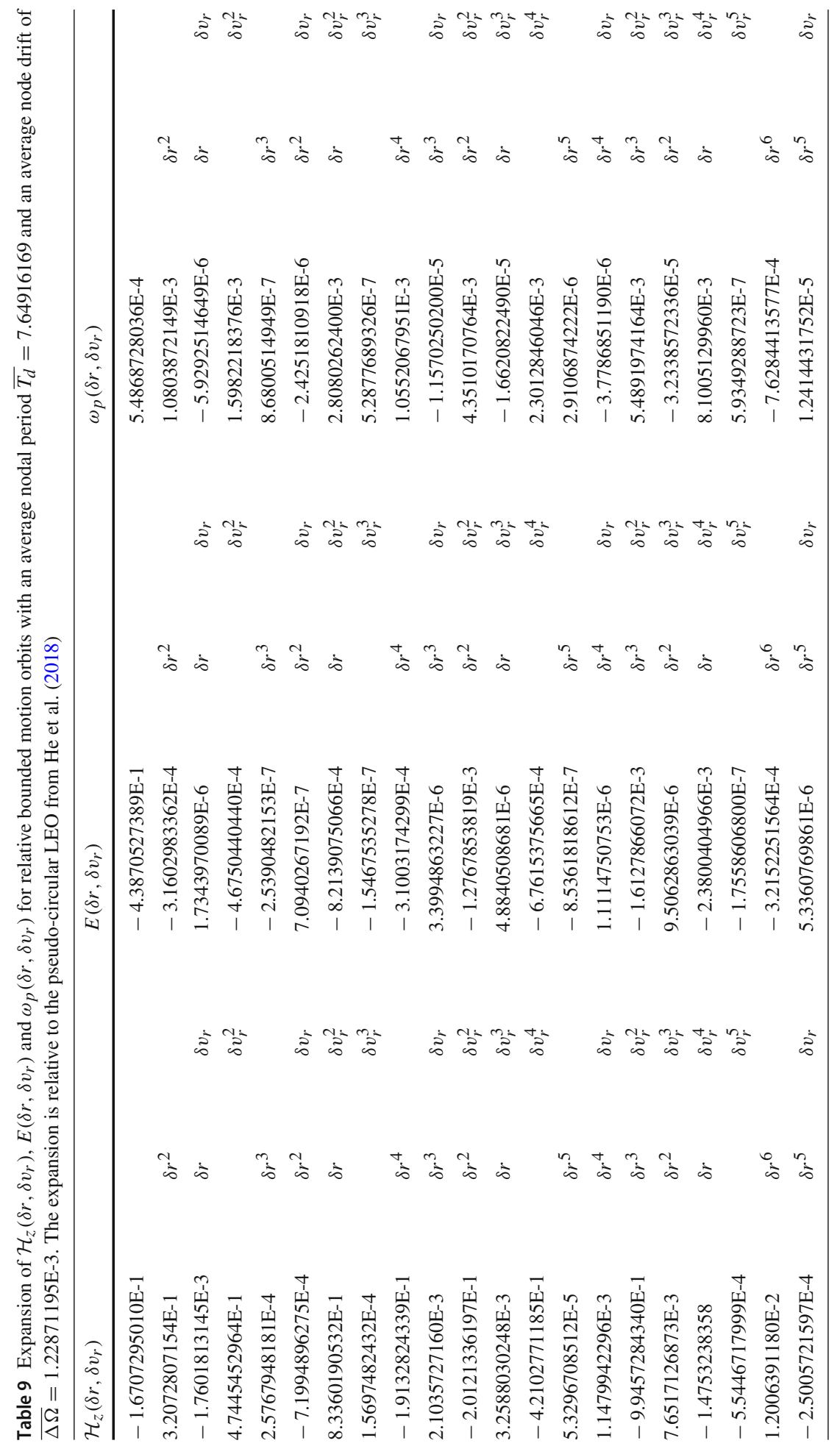




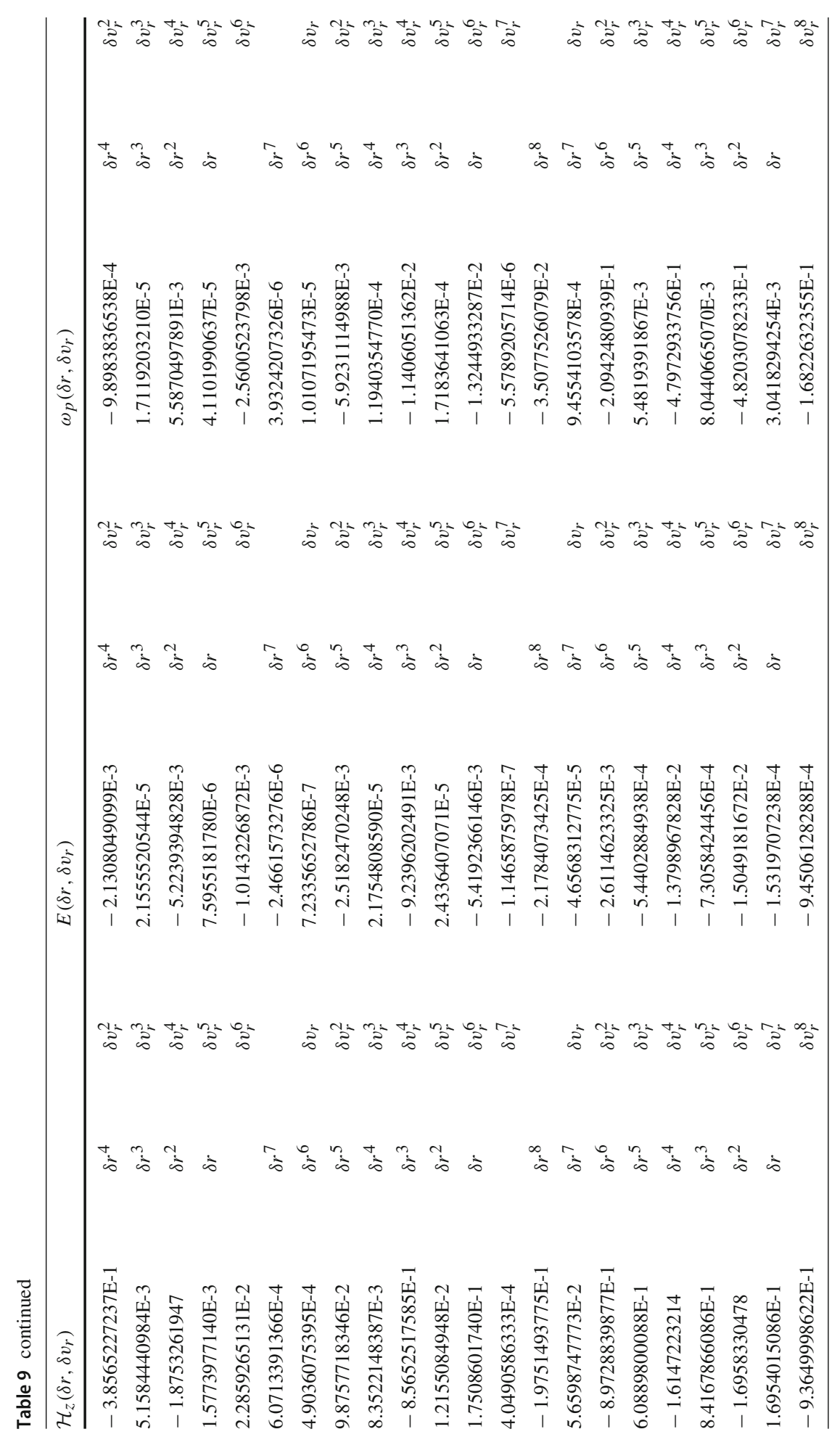




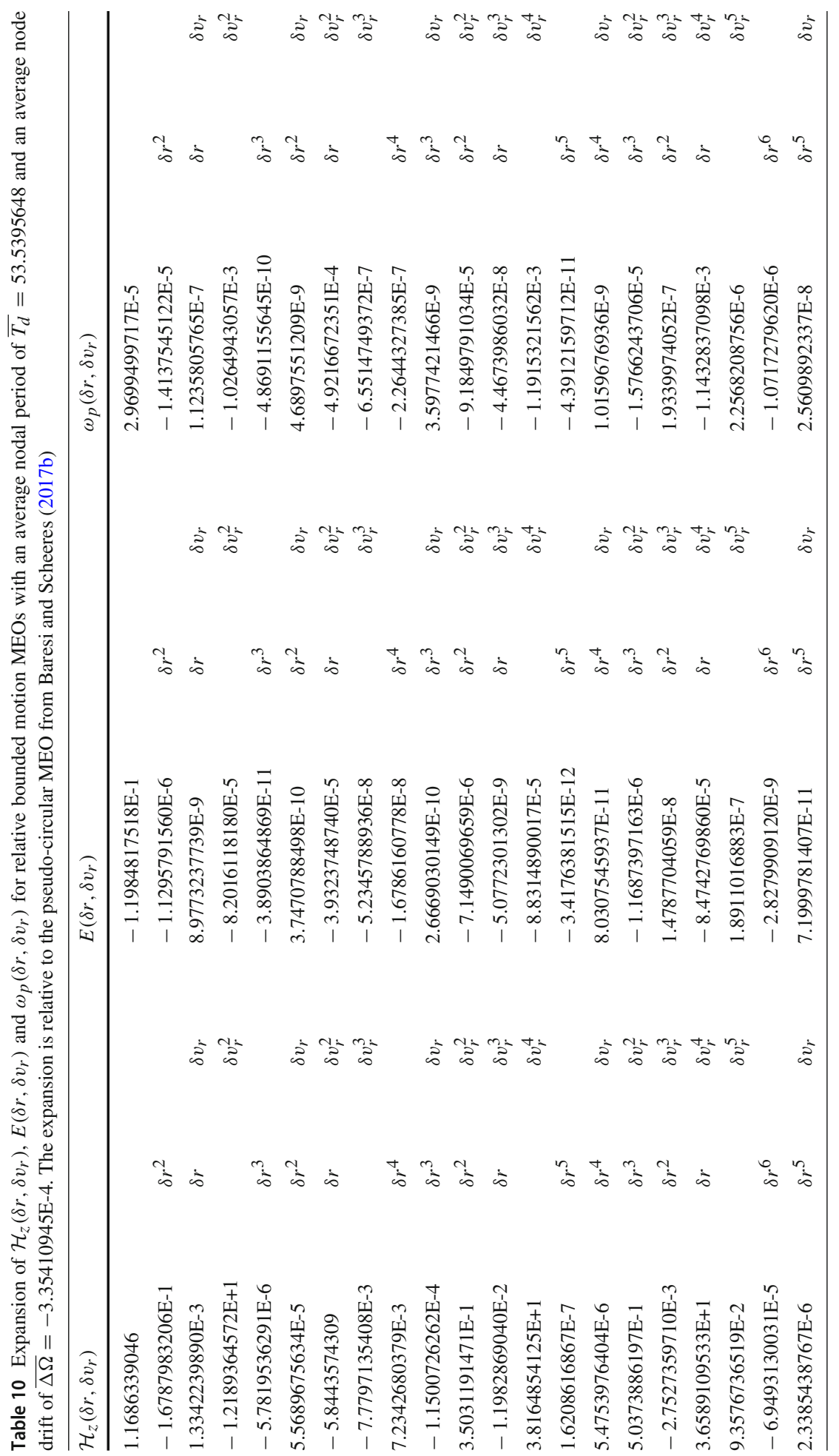




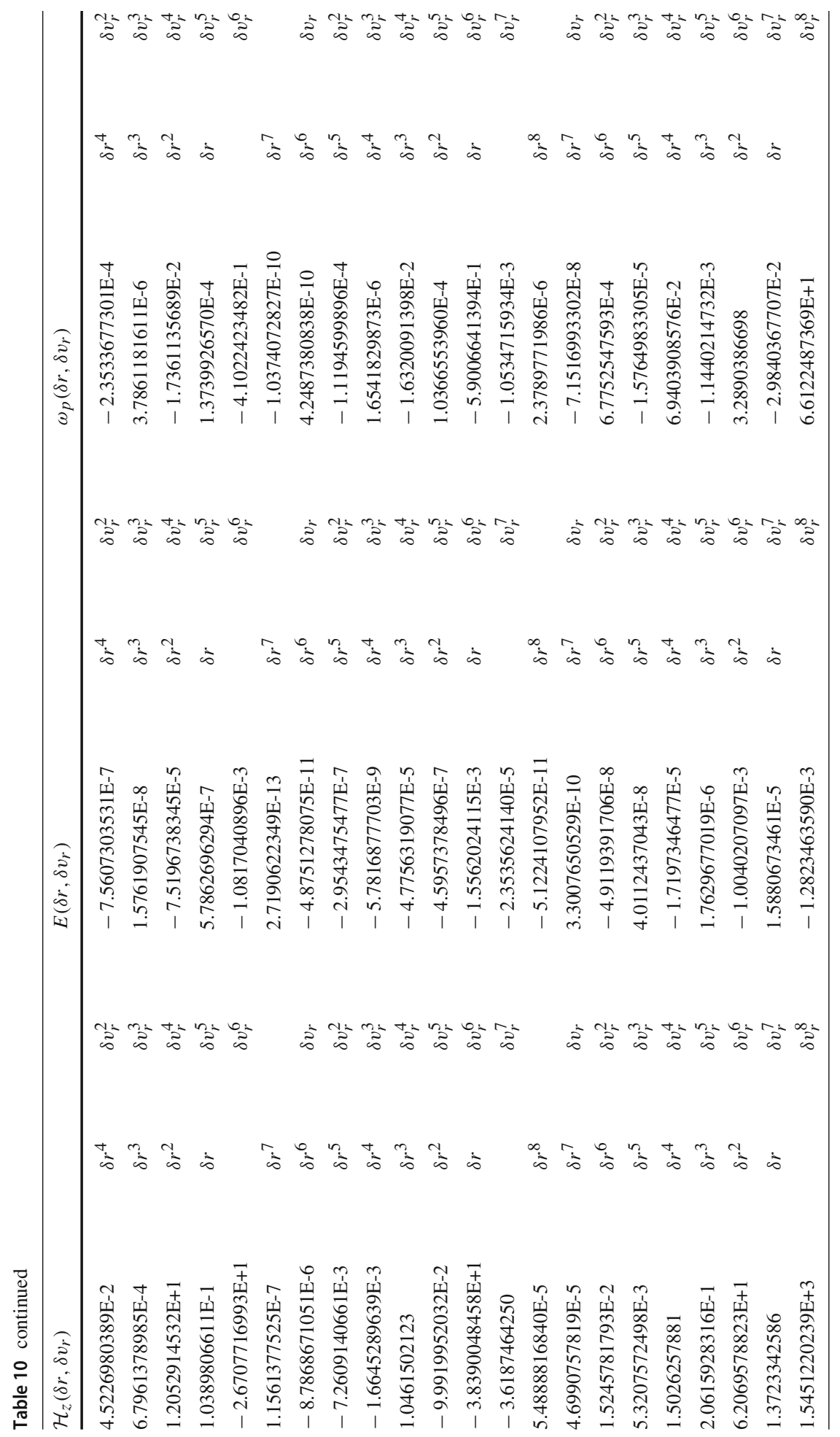




\section{References}

Alfriend, K.T., Vadali, S.R., Gurfil, P., How, J.P., Breger, L.S.: Spacecraft Formation Flying: Dynamics, Control and Navigation, pp. 144-146. Butterworth-Heinemann, Oxford (2010)

Armellin, R., Di Lizia, P., Berz, M., Makino, K.: Computing the critical points of the distance function between two Keplerian orbits via rigorous global optimization. Celest. Mech. Dyn. Astron. 107(3), 377-395 (2010)

Armellin, R., Di Lizia, P., Topputo, F., Lavagna, M., Bernelli-Zazzera, F., Berz, M.: Gravity assist space pruning based on differential algebra. Celest. Mech. Dyn. Astron. 106(1), 1-24 (2010)

Baresi, N., Olikara, Z.P., Scheeres, D.J.: Fully numerical methods for continuing families of quasi-periodic invariant tori in astrodynamics. J. Astron. Sci. (2018). https://doi.org/10.1007/s40295-017-0124-6

Baresi, N., Scheeres, D.J.: Bounded relative motion under zonal harmonics perturbations. Celest. Mech. Dyn. Astron. 127(4), 527-548 (2017). https://doi.org/10.1007/s10569-016-9737-5

Baresi, N., Scheeres, D.J.: Design of bounded relative trajectories in the earth zonal problem. J. Guid. Control Dyn. 40(12), 3075-3087 (2017). https://doi.org/10.2514/1.G002603

Berz, M.: The method of power series tracking for the mathematical description of beam dynamics. Nucl. Instrum. Methods A 258(3), 431-436 (1987). https://doi.org/10.1016/0168-9002(87)90927-2

Berz, M.: Differential algebraic description of beam dynamics to very high orders. Part. Accel. 24(SSC-152), 109-124 (1988)

Berz, M.: Differential algebraic formulation of normal form theory. In: Conference series-Institute of Physics, vol. 131, pp. 77-86. IOP Publishing LTD (1993)

Berz, M.: Modern Map Methods in Particle Beam Physics. Academic Press, Cambridge (1999)

Berz, M., Makino, K.: COSY INFINITY Version 10.0 beam physics manual. Technical report MSUHEP151103-rev, Michigan State University, East Lansing (2017)

Broucke, R.A.: Numerical integration of periodic orbits in the main problem of artificial satellite theory. Celest. Mech. Dyn. Astron. 58(2), 99-123 (1994). https://doi.org/10.1007/BF00695787

Brown, O., Eremenko, P.: Fractionated space architectures: a vision for responsive space. Technical report Defense Advanced Research Projects Agency Arlington VA (2006)

Chu, J., Guo, J., Gill, E.K.A.: Long-term passive distance-bounded relative motion in the presence of $J_{2}$ perturbations. Celest. Mech. Dyn. Astron. 121(4), 385-413 (2015). https://doi.org/10.1007/s10569-0159603-x

Clohessy, W.H., Wiltshire, R.S.: Terminal guidance system for satellite rendezvous. J. Aerosp. Sci. 27(9), 653-658 (1960). https://doi.org/10.2514/8.8704

Cook, G.E.: Luni-solar perturbations of the orbit of an earth satellite. Geophys. J. Roy. Astron. Soc. 6(3), 271-291 (1962). https://doi.org/10.1111/j.1365-246X.1962.tb00351.x

D'Amico, S., Ardaens, J.S., Larsson, R.: Spaceborne autonomous formation flying experiment on the PRISMA mission. J. Guid. Control Dyn. 35(3), 834-850 (2012). https://doi.org/10.2514/1.55638

D'Amico, S., Montenbruck, O.: Proximity operations of formation-flying spacecraft using an eccentricity/inclination vector separation. J. Guid. Control Dyn. 29(3), 554-563 (2006). https://doi.org/10.2514/ 1.15114

Dang, Z., Wang, Z., Zhang, Y.: Improved initialization conditions and single impulsive maneuvers for $J_{2}$ invariant relative orbits. Celest. Mech. Dyn. Astron. 121(3), 301-327 (2015). https://doi.org/10.1007/ s10569-014-9601-4

Gim, D.W., Alfriend, K.T.: State transition matrix of relative motion for the perturbed noncircular reference orbit. J. Guid. Control Dyn. 26(6), 956-971 (2003). https://doi.org/10.2514/2.6924

Grote, J., Berz, M., Makino, K.: High-order representation of Poincaré maps. Nucl. Instrum. Methods A 558(1), 106-111 (2006). https://doi.org/10.1016/j.nima.2006.01.001

Gurfil, P.: Relative motion between elliptic orbits: generalized boundedness conditions and optimal formationkeeping. J. Guid. Control Dyn. 28(4), 761-767 (2005). https://doi.org/10.2514/1.9439

Gurfil, P.: Generalized solutions for relative spacecraft orbits under arbitrary perturbations. Acta Astronaut. 60(2), 61-78 (2007). https://doi.org/10.1016/j.actaastro.2006.07.013

He, Y., Armellin, R., Xu, M.: Bounded relative orbits in the zonal problem via high-order Poincaré maps. J. Guid. Control Dyn. 42(1), 91-108 (2018). https://doi.org/10.2514/1.G003612

Inalhan, G., Tillerson, M., How, J.P.: Relative dynamics and control of spacecraft formations in eccentric orbits. J. Guid. Control Dyn. 25(1), 48-59 (2002). https://doi.org/10.2514/2.4874

Kolchin, E.R.: Differential Algebra and Algebraic Groups, vol. 54. Academic press, Cambridge (1973)

Koon, W.S., Marsden, J.E., Murray, R.M., Masdemont, J.: $J_{2}$ dynamics and formation flight. In: AIAA Guidance, Navigation, and Control Conference and Exhibit. AIAA, Montreal, Canada (2001)

Lizia, P.D., Armellin, R., Lavagna, M.: Application of high order expansions of two-point boundary value problems to astrodynamics. Celest. Mech. Dyn. Astron. 102(4), 355-375 (2008). https://doi.org/10.1007/ s10569-008-9170-5 
Makino, K., Berz, M.: COSY INFINITY version 9. Nucl. Instrum. Methods 558(1), 346-350 (2006). https:// doi.org/10.1016/j.nima.2005.11.109

Martinusi, V., Gurfil, P.: Closed-form solutions for satellite relative motion in an axially-symmetric gravitational field. Adv. Astronaut. Sci. 140, 1525-1544 (2011)

Montenbruck, O., Kirschner, M., D’Amico, S., Bettadpur, S.: E/I-vector separation for safe switching of the grace formation. Aerosp. Sci. Technol. 10(7), 628-635 (2006). https://doi.org/10.1016/j.ast.2006.04.001

Poincaré, H.: Les méthodes nouvelles de la mécanique céleste, vol. I-III. Gauthier-Villars it fils, Paris (1899)

Ritt, J.F.: Differential equations from the algebraic standpoint, vol. 14. American Mathematical Soc, Washington, D.C. (1932)

Ritt, J.F., Liouville, J.: Integration in finite terms: Liouville's theory of elementary methods. Columbia University Press, New York (1948)

Schaub, H., Alfriend, K.T.: $J_{2}$ invariant relative orbits for spacecraft formations. Celest. Mech. Dyn. Astron. 79(2), 77-95 (2001). https://doi.org/10.1023/A:1011161811472

Schweighart, S.A., Sedwick, R.J.: High-fidelity linearized J model for satellite formation flight. J. Guid. Control Dyn. 25(6), 1073-1080 (2002). https://doi.org/10.2514/2.4986

Sullivan, J., Grimberg, S., D’Amico, S.: Comprehensive survey and assessment of spacecraft relative motion dynamics models. J. Guid. Control Dyn. 40(8), 1837-1859 (2017). https://doi.org/10.2514/1.G002309

Vadali, S., Schaub, H., Alfriend, K.: Initial conditions and fuel-optimal control for formation flying of satellites. In: AIAA Guidance, Navigation, and Control Conference and Exhibit. Portland, OR (1999)

Weisskopf, A.: Introduction to the differential algebra normal form algorithm using the centrifugal governor as an example. Technical Report MSUHEP-190617, Department of Physics and Astronomy, Michigan State University, East Lansing (2019). arXiv:1906.10758 [physics.class-ph]

Weisskopf, A., Tarazona, D., Berz, M.: Computation and consequences of high order amplitude- and parameterdependent tune shifts in storage rings for high precision measurements. Int. J. Mod. Phys. A 34, 1942011 (2019). https://doi.org/10.1142/S0217751X19420119

Wittig, A., Di Lizia, P., Armellin, R., Makino, K., Bernelli-Zazzera, F., Berz, M.: Propagation of large uncertainty sets in orbital dynamics by automatic domain splitting. Celest. Mech. Dyn. Astron. 122(3), 239-261 (2015). https://doi.org/10.1007/s10569-015-9618-3

$\mathrm{Xu}, \mathrm{M}$., Wang, Y., Xu, S.: On the existence of $J_{2}$ invariant relative orbits from the dynamical system point of view. Celest. Mech. Dyn. Astron. 112(4), 427-444 (2012). https://doi.org/10.1007/s10569-012-9401-7 